\title{
Probing the prediction of effective properties for composite materials
}

\author{
K.B. Shingare, S. Naskar* \\ Department of Aerospace Engineering, Indian Institute of Technology Bombay, Mumbai, India
}

\section{A R T I C L E I N F O}

\section{Keywords:}

Representative volume element

Finite element (FE) methods

Composite materials

Micromechanical models

Piezoelectric materials

Homogenization

\begin{abstract}
A B S T R A C T
This article presents different micromechanical modelling techniques based on analytical and numerical approaches to determine the effective elastic and piezoelectric (piezoelastic) properties of graphene-based composite materials. Different types, orientations and shapes as well as different geometrical parameters of fiber reinforcement are considered for estimating the effective properties. The effective properties of composite are predicted with and without considering the strong covalent bond which provides interaction and in-plane stability of 2Dcrystalline graphene or strong van der Wall forces formed between graphene layers and the matrix. It is revealed that the axial, transverse and shear effective piezoelastic properties of graphene reinforced piezoelectric composite (GRPC) are significantly enriched due to the incorporation of graphene into the epoxy matrix. The importance of incorporating graphene as nanofillers/interphase into the conventional epoxy matrix to form an advanced composite and its effective properties are illustrated while these results show excellent agreement with previously reported experimental estimates. These results reveal that due to incorporation of graphene nanofillers, there is a significant enhancement in effective properties of composite. The results would also help to recognize the most important material properties with respect to different shapes and orientation of reinforcements which influences the performance of system significantly. To confirm safety, robustness and sustainability of the structure, it is the most prior requirement to determine the effective properties of composites considering different parameters for the different static and structural analyses.
\end{abstract}

\section{Introduction}

During past couple of decades, carbon-based materials such as carbon nanotubes (CNTs)- and graphene-reinforced composites are broadly used in several fields of applications such as aerospace, mechanical, automotive, civil as well as marine industries. Novoselov et al. (2004) discovered an exceptional single atomic layered two-dimensional graphene which is extracted from three-dimensional graphite using "Scotch Tape" method. The global interest in this "novel material" is still growing, which can be observed from the huge investment and also from the increasing new developments per year in scientific research. Due to its high electro-thermo-mechanical properties such as electrical conductivity $(\sim 6000 \mathrm{~S} / \mathrm{m}$ ) (Du et al., 2008), thermal conductivity $(\sim 5000 \mathrm{~W} / \mathrm{mk})$, elastic modulus $(\sim 1 \mathrm{TPa})$ along with this it is having large surface area $\left(2630 \mathrm{~m}^{2} / \mathrm{g}\right)$ and electron mobility $\left(\sim 250,000 \mathrm{~cm}^{2} / \mathrm{Vs}\right)$ (Park and Ruoff, 2009) at room temperature and these all properties make it striking for use in various engineering applications. Hence, the study of the electromechanical properties of graphene-reinforced composites (GRCs) became a huge area of research in academia as well as industry. For effective use of such weight-sensitive composite, a prudent understanding of its static and dynamic characteristics are important. At the same time, it is also essential to accurately determine the effective properties and parameters affecting these properties such as different shapes of fiber inclusions. In the past, several researchers (Hagood and Bent, 1993; Berger et al., 2007; Esteva and Spanos, 2009) performed investigations on the effective properties of different shapes and types of reinforced composites. The increase in demand of lightweight, high strength materials in automobile and aerospace industries invites the researcher to use advanced technology for the development of new multifunctional engineering materials with superior mechanical and thermal properties that were not found in conventional materials. Polymer nanocomposites are excellent structural engineering materials, which have lightweight and desired functional properties and it can be used in a wide variety of engineering and industrial applications. Graphene-based composite materials have the potential to use as chemical sensors because of their extraordinary sensitivity. These outcomes suggest, GRCs materials are the perfect replacements for micro- and nano-electromechanical system

\footnotetext{
* Corresponding author.

E-mail address: susmitanaskar@iitb.ac.in (S. Naskar).
} 
(M-/NEMS) applications. Specifically, GRCs with good semiconducting properties, which makes it suitable to produce strain sensor having a high gauge factor. Hence, the electromechanical properties of GRCs are becoming increasingly popular in many design and functional engineering requirements.

Over the years, the prediction of effective properties using analytical, semi-analytical and finite element (FE) methods based on different assumptions such as homogeneous, parallel and continuous reinforced fiber, no slippage condition between fiber and matrix, uniform stresses and strains conditions have been carried out by several researchers (Voigt, 1910; Reuss, 1929; Halpin and Thomas, 1968; Smith and Auld, 1991; Benveniste and Dvorak, 1992; Berger et al., 2005). The pioneering work in this research field was carried out by, Voigt (1910) and Reuss (1929), who derived average methods for polycrystals by assuming the condition of uniform stress or strain of the specimen. These procedures define lower and upper bounds for elastic moduli of polycrystals that was proposed by Hill (1952). Afterward, based on variational principles, Hashin and Shtrikman (1962a, 1962b) derived models with more accurate bounds of elastic modules. They considered the representative volume element (RVE), which was extracted from periodic heterogeneous composite material and were very identical to "polycrystal cubical element". While considering the periodic boundary conditions (PBCs), Suquet (1987) proposed that the uniform strains/stresses conditions at the boundaries are not a better option for this type of RVE because these uniform stresses underestimate material properties while overestimated by uniform strains. Therefore, the significant outcomes are attained by applying PBCs to an RVE. To obtain suitable constitutive model at macroscopic level, many homogenization techniques (Suquet, 1987; Yang and Qin, 2001; Sun and Tzeng, 2002; Greco and Luciano, 2011) were proposed which is based on the concept of mechanics that transforms a body of a heterogeneous material into small body of a homogeneous material. The macroscopic properties are estimated by such a homogenization technique, which produces the effective stresses and strains acting on the effective small homogenized material which is called RVE. Such small RVE represents the properties equivalent to that of its composite lamina because the strain energy stored in both is nearly equal. By using the similar idea of RVE in conjunction with the FE numerical methods, many researchers (Yang and Qin, 2001; Sun and Tzeng, 2002; Berger et al., 2005; Kari et al., 2007; Rodríguez-Ramos et al., 2013; Tian et al., 2015; Martínez-Ayuso et al., 2017) used such a powerful method to predict effective properties including elastic, piezoelectric as well as dielectric constants considering the concept of single RVE. Several authors (Chandra et al., 2012; Patil et al., 2017; Mirabedini et al., 2020; Kumar et al., 2019) investigated the response of the composite materials with multiscale approach. The overall properties of the composites which are estimated through the experimentation and are well fitted with the investigations performed through the micro-/macro-mechanical analysis. Due to lack of structural simplicity of aligned/continuous fiber-reinforced composite laminates, the FE method is widely used as compared to analytical modelling for multiphase materials. Hence, to evaluate the effective properties of multi-scaled or multi-shaped fiber composites, FE method is well-suited technique. Luciano and Tamburrino (2000) obtained the homogenized dielectric constitutive equations for heterogeneous materials characterized by a random arrangement of the inclusions with the help of Hashin and Shtrikman variational principle considering macroscopic charge density. Several illustrations of overall constitutive equations are also developed for random and periodic composites and presented comparisons with exact findings for laminates. Yang and Qin (2001) studied the effective elastoplastic properties of metal matrix composite reinforced by short fiber through micromechanics and FE model by assuming fiber and matrix as elastic and elasto-visco-plastic continuum medium, respectively. They found good agreement with experimental estimates for effective elastoplastic properties of boron/aluminum composites. Using the same concept of RVE, Kari et al. (2007) evaluated the effective material properties of composites reinforced with spherical particles for distinct volume fractions and also considered the effect of the size of spherical particles on it. Greco and Luciano (2011) developed the numerical and theoretical nonlinear analysis of the homogenized response of composite with periodic microstructure exposed to macroscopic constant strain by considering the effects of instabilities arising at microscopic and macroscopic scales. Based on analytical formulation of asymptotic homogenization method (AHM), Rodríguez-Ramos et al. (2013) considered two-phase piezoelectric composites to predict their effective properties whereas their individual phases are transversely isotropic and the unit cells have different shapes. By means of different parallelogram cells and linear spring model, the imperfect mechanical contact at the interface of the composites has also been assumed.

Recently researchers (Patil et al., 2017; Deierling and Zhupanska, 2018; Gopinath and Batra, 2018; Zhu et al., 2018; Zhou et al., 2020; Mukhopadhyay et al., 2016) investigated the effective properties of composites using methods based on homogenization and RVE/unit cell technique. Patil et al. (2017) predicted the effective properties of heterogeneous materials by applying the extended as well as multiscale FE method (XFEM and MsFEM) in which the particles and voids were incorporated into the matrix. They have considered three types of voids and particles incorporated in matrix such as voids, hard particles as well as both voids and hard particles, and determined the effective properties by applying PBCs to the RVE. Deierling and Zhupanska (2018) developed the FE- and RVE-based models to estimate the effective thermoelastic properties of the spatial variation of graded multiphase particulate reinforced composites. To generate the high-resolution with spatial variation of graded microstructures of the constituents of composites, the effective particle packing algorithms were used. Whereas, to determine the effective elastic stiffness coefficients, Zhu et al. (2018) presented a homogenization-based hybrid uncertain analysis method considering undefined but confined parameters. The coefficients with these undefined parameters can be expanded by using the Gegenbauer series expansion method using the FE homogenization technique. Gopinath and Batra (2018) formulated three micromechanical models such as Fourier series, method of cells and transformation field analysis to obtain the final expressions related to the local sub-cell strain to the homogenized macro-strain in a unit cell are same. They estimated the stresses and strains in each sub-cell from the stiffnesses and macro-strains by using these micromechanical models. Pingaro et al. (2019a, 2019b) studied the computational homogenization technique and adopted a statistical method for the definition of the RVE size, that in random media to predict effective elastic moduli. Based on the virtual element method, they also proposed a fast-statistical homogenization (FSH) technique developed within unified framework that presets all the phases to perform. Numerous simulations were carried out by changing the intensity of porosity, emphasizing the effectiveness of FSH with virtual elements of degree one. Fantuzzi et al. (2020) modelled the woven fabrics as orthotropic interlaced yarns within a polymer matrix which can have additives. For this instance, carbon nanotubes are considered as reinforcement additives in the polymer matrix which gives enhanced mechanical properties of composite. They also compared the analytical and experimental mechanical properties of carbon woven textile composites. Zhou et al. (2020) proposed a new RVE modelling technique for woven composite considering large volume fraction of yarn, constant mesh and geometrical parameters. They also investigated the mechanical properties and damage analysis of woven fabric composite at the mesoscale level when subjected to in-plane tension/compression through experimentation and simulation. Naskar and co-authors (Naskar et al., 2017, 2018, 2019) proposed new micromechanical analysis for predicting effective properties of fiber-reinforced composite and functionally graded material (FGM) by using novel approach of stochastic representative volume element (SRVE) considering spatial distribution with stochastic analysis and uncertainty quantifications (UQ)..

Apart from these studies, interphase/nanofillers is a complex region that can be used in-between the fiber reinforcement and matrix to obtain more improved effective properties of composite materials. To obtain more precise and accurate composite properties, the perfect bonding (no slippage condition) hypothesis is usually considered between the fiber, interphase and 
matrix. For this instance, to illustrate the thermal conductivity of polymer nanocomposites, Shin et al. (2013) proposed the multiscale homogenization technique for consideration of the Kapitza thermal resistance at the interface and the polymer immobilized interphase. Chen et al. (2014) studied gradient interphase embedded by graphene oxide (GO) in the composites to enhance the distribution of carbon fiber (CF)/epoxy interface as well as the stress transfer characteristics. Therefore, in recent advances, the novel graphene or its derivatives can be used as nanofillers as well as interphase to improve the mechanical properties of composites. Yamaguchi et al. (2017) studied the effect of silica nanofiller particle diameters in a computer-aided design/manufacturing composite resin block on physical properties at themultiscale in silico. These blocks were modelled with different sizes of silica nanofiller particles and matrix to predict Poisson's ratios and Young's moduli at the macro-scale using homogenization. Mirabedini et al. (2020) proposed the integration of graphene for developing strategies of fabricating multiscale graphene enhanced fiber-reinforced polymer composites and its functional properties that allow for innovative and stimulating smart structural applications. Through a single-step improved chemical vapor deposition (CVD) technique without any catalyst, Karakassides et al. (2020) investigated radially aligned graphene nanoflakes which is used as a novel nano-reinforced interphase directly grown on CFs. On careful examining the available literature, it shows that there is no single study available on piezoelectric composite decorated or incorporated with graphene interphase to improve its electromechanical properties. Hence it is crucial to investigate the micromechanical modelling for graphene-based composite materials.

To the authors' best knowledge, this novel graphene-reinforced piezoelectric composites (GRPCs) which give numerous opportunities for developing MEMS/NEMS has not been conveyed hitherto which can act as a replacement for the traditional piezoelectric nanostructure applications. This is an undeniable encouragement behind this novel work. In order to establish these novel GRCs and GRPCs as the superior advanced composite for structural applications, static/dynamic analysis must be carried out using this composite material and for such analysis, all effective properties of these composites must be known a priori. Due to the absence of structural simplicity of parallel/continuous fiber composite, the FE numerical techniques are frequently used as compared to analytical modelling for multiphase materials. Specifically, the current novel work deals with (i) different micromechanical models including rules-of-mixture (ROM/mROM), Halpin-Tsai (HT) model, two- and three-phase micromechanical model based on mechanics of materials (MOM) approach to determine the elastic and piezoelectric properties of GRPC in-conjunction with short glimpse of graphenereinforced FGM for future studies, (ii) FE numerical models to validate the results obtained from micromechanical model and from existing experimental studies for GRC and (iii) effect of different shapes (continuous, cylindrical and ellipsoidal) of graphene nanofillers using 2D and 3D RVE by applying PBCs on the effective properties of the composite. Most significantly, the present findings reveal that the introduction of graphene as nanofillers/interphase significantly influences the effective properties of GRPC compared to that of the conventional piezoelectric composite. This is clearly illustrated by comparison of distinct sets of results such as (i) conventional GRC (without piezoelectric fiber), (ii) PZT/epoxy composites (PRC - without graphene interphase) and (iii) GRPC (with graphene interphase and piezoelectric fiber) herein. Finally, the present research work fulfils a gap in our knowledge based on considering the effect of piezoelectricity as well as graphene interphase.

\section{Micromechanical models}

In the present study, different micromechanical models based on an analytical approach are developed to determine the effective properties including elastic and piezoelectric properties of composite lamina and laminates with different types and shapes of fibers and inclusions. Several researchers (Rafiee et al., 2009; Young et al., 2012; Li et al., 2018) used the micromechanical models like rules-of-mixture (ROM), modified rules-of-mixture (mROM), shear-lag and Halpin-Tsai (HT) models for investigating the mechanical behavior of composites and also validated with experimental studies which were carried out for graphene-reinforced composite (GRC). Hence, to predict the properties of nanocomposite, it is essential to consider the nanofiber and matrix, which are subjected to either iso-strain or iso-stress condition in the proposed composite.

\subsection{Estimation of volume/weight fraction}

The functionally graded material (FGM) is a type of composite material which is inhomogeneous mixture of two materials (Kashtalyan, 2004; Woodward and Kashtalyan, 2011; Dey et al., 2015a, 2015b, 2019; Mukhopadhyay et al., 2018, 2020; Karsh et al., 2019; Dey et al., 2016). In recent studies, most of the researchers (Ansari et al., 2019; Wu et al., 2017; Zhang et al., 2020) fabricated and investigated these FGMs by reinforcing graphene and its derivatives into various types of matrices. In case of FGMs, volume/weight fractions of composite lamina are achieved by using different patterns such as: (i) unidirectional (UD), (ii) X, (iii) O and (iv) A type (Zhang et al., 2020). In case of UD-pattern, the graphene content remains same in all layers which resemble to a homogeneous composite. The graphene volume fraction differs from layer to layer such as the first and last layers of composite are graphene rich in $\mathrm{X}$-pattern while the mid-layers are graphene rich in O-pattern. The graphene volume fraction rises from the first to the last layer in A-pattern. If the FG multi-layered GRC are considered with an even number of layers. The volume fractions $\left[V_{g}(k)\right]$ of each individual $\left(k^{\text {th }}\right)$ layer for the four distribution patterns can be described as following (Wu et al., 2017; Zhang et al., 2020):

$$
\begin{aligned}
& \mathrm{UD} \text { pattern }-V_{g}(k)=V_{g}^{*} \\
& \mathrm{X}-\text { pattern }-V_{g}(k)=2 V_{g}^{*}(|2 k-N-1| / N) \\
& \mathrm{O}-\text { pattern }-V_{g}(k)=2 V_{g}^{*}(1-|2 k-N-1| / N) \\
& \mathrm{A}-\text { pattern }-V_{g}(k)=V_{g}^{*}((2 k-1) / N)
\end{aligned}
$$

where $V_{g}^{*}=\frac{w_{g}}{w_{g}+\left(\rho_{g} / \rho_{m}\right)\left(1-w_{g}\right)}$ and $\rho_{g}$ and $\rho_{m}$ denote the density of graphene nanofillers and epoxy matrix, $w_{g}$ denotes the weight fraction of whole composite and $N$ indicates the number of layers in composite. The volume fractions of each layer of FGM using different patterns are enlisted in Table 1. By using different micromechanical model, one can determine the effective properties of each layer of FGM.

\subsubsection{Rules of mixture (ROM)}

One of the most widely and simple expression which was developed to describe the reinforcement achieved from a high-modulus filler in a low-modulus matrix, is known as "rule of mixtures", in which the effective elastic modulus of the composite can be written as follows (Young and Lovell, 2011):

Table 1

Volume fraction of each layer of GRC (FGM) for different patterns.

\begin{tabular}{lllll}
\hline \multirow{2}{*}{ Layer No. } & \multicolumn{4}{l}{ Volume fraction of 10 layers } \\
\cline { 2 - 5 } & $\mathrm{U}$ & $\mathrm{X}$ & $\mathrm{O}$ & $\mathrm{A}$ \\
\hline 1 & 0.11007 & 0.19813 & 0.02201 & 0.01101 \\
2 & 0.11007 & 0.1541 & 0.06604 & 0.03302 \\
3 & 0.11007 & 0.11007 & 0.11007 & 0.05504 \\
4 & 0.11007 & 0.06604 & 0.1541 & 0.07705 \\
5 & 0.11007 & 0.02201 & 0.19813 & 0.09907 \\
6 & 0.11007 & 0.02201 & 0.19813 & 0.12108 \\
7 & 0.11007 & 0.06604 & 0.1541 & 0.1431 \\
8 & 0.11007 & 0.11007 & 0.11007 & 0.16511 \\
9 & 0.11007 & 0.1541 & 0.06604 & 0.18713 \\
10 & 0.11007 & 0.19813 & 0.02201 & 0.20914 \\
\hline
\end{tabular}


$E_{c}=E_{f} v_{f}+E_{m} v_{m}$

where $E_{f}, E_{m}$ and $E_{c}$ denote the elastic modulus of fiber, matrix and composite while $v_{f}$ and $v_{m}$ denote volume fraction of fiber and matrix. The Eq. (5) gives better results for low volume fraction of fibers in composite while in case of higher volume fraction, this relation does not provide good estimates because of some essential/unavoidable factors such as orientations, agglomeration and efficiency factor of reinforcement which are not taken into considerations (Guzmán de Villoria and Miravete, 2007).

\subsubsection{Modified rules of mixture (mROM)}

As the conventional ROM technique doesn't consider the factors such as the orientation of fillers, length and agglomeration of nanofibers while these factors are important in case of nanocomposite and taken into consideration using the modified rules of mixture (mROM) by taking suitable Krenchel orientation factor $\left(\eta_{0}\right)$, critical length efficiency factor $\left(\eta_{l}\right)$ and agglomeration factor $\left(\eta_{a}\right)$ (Guzmán de Villoria and Miravete, 2007; Young et al., 2012). This model is very similar to the Cox model (Young et al., 2012) for composite reinforced with random short fibers. Hence, by considering all these factors, the expression for ROM can be rewritten as (Li et al., 2018):

$$
E_{c}=\eta_{0} \eta_{l} \eta_{a} E_{f} v_{f}+E_{m} v_{m}
$$

Using mROM technique, we can calculate the effective modulus of GRC for the uniform distribution of aligned/parallel or continuous and random nanofiber (showed in Fig. 1) by assuming the perfect bond between the fiber and matrix, and the matrix should be free from voids. Moreover, one can also determine the effective properties of composite considering two-dimensional (2D), three-dimensional (3D)fiber reinforcement as well as aligned and random orientation of fibers with and without considering agglomeration. But these traditional methods are unable to predict all effective properties of graphene reinforced piezoelectric composite (GRPC) such as effective elastic and piezoelectric properties including transverse and shear coefficients. Therefore, a micromechanics model based on assumptions of simple ROM and isostress/iso-strain conditions is used to predict the effective properties of GRC and GRPC assuming graphene nano-fillers as continuum/microscale fibers embedded into the epoxy matrix. Using the two- and three-phase micromechanics model, all effective properties of piezoelectric composite are estimated by assuming above all consideration regarding the individual phase of composite and presented in next section 2.1.4.

\subsubsection{Halpin-Tsai (HT) model}

In case of ROM and mROM, besides the different orientation, agglomeration or critical length factor, it did not consider the geometrical parameters such as length, width, thickness and diameter of reinforced fiber. Therefore, these geometrical parameters are considered while determining the effective properties of the nanocomposites by using the well-known Halpin-Tsai (HT) model (Guzmán de Villoria and Miravete, 2007; Zhang et al., 2020). The graphene-based nanocomposites are assumed (Qin et al., 2020) by introducing the graphene or its different derivatives into epoxy matrix; the modulus of the composite can be formulated as follows:

$E_{c}=\frac{3}{8} E_{L}+\frac{5}{8} E_{T}$

where,

$E_{L}=\frac{1+\xi_{L} \eta_{L} v_{g}}{1+\eta_{L} v_{g}} E_{m}, \quad E_{T}=\frac{1+\xi_{T} \eta_{T} v_{g}}{1+\eta_{T} v_{g}} E_{m}$

whereas, the parameter $\xi$ changes with respect to the boundary condition and geometry of the fiber. The detailed descriptions of these parameters for rectangular shaped fiber are defined by Halpin and Thomas (1968), Halpin (1969) and Affdl and Kardos (1976) and can be written as following:

$\eta_{L}=\frac{\left(E_{g} / E_{m}\right)-1}{\left(E_{g} / E_{m}\right)+\xi_{L}}, \eta_{T}=\frac{\left(E_{g} / E_{m}\right)-1}{\left(E_{g} / E_{m}\right)+\xi_{T}}$

$\xi_{L}=2\left(a_{g} / t_{g}\right), \quad \xi_{T}=2\left(b_{g} / t_{g}\right)$

$\xi_{L}$ can be re-expressed as:

$\xi_{L}=2\left(a_{g} / t_{g}\right)\left(b_{g} / t_{g}\right)$

where $a_{g}, b_{g}$ and $\mathrm{t}_{g}$ denote the width, length and thickness of graphene nanoplatelets or any fiber while $d_{g}$ denotes the diameter of fiber.

\subsubsection{Mechanics of material (MOM) model}

2.1.4.1. Two- and three-phase model. In this section, the effective piezoelastic properties of GRPC are obtained by deriving two- and threephase micromechanical models. The difference between the two- and three-phase model is nothing but the model derived considering how many numbers of individual phases. If we neglect the interphase phase between the fiber and matrix, the model can be called a two-phase model; otherwise, it can be called as a three-phase model. Here, the
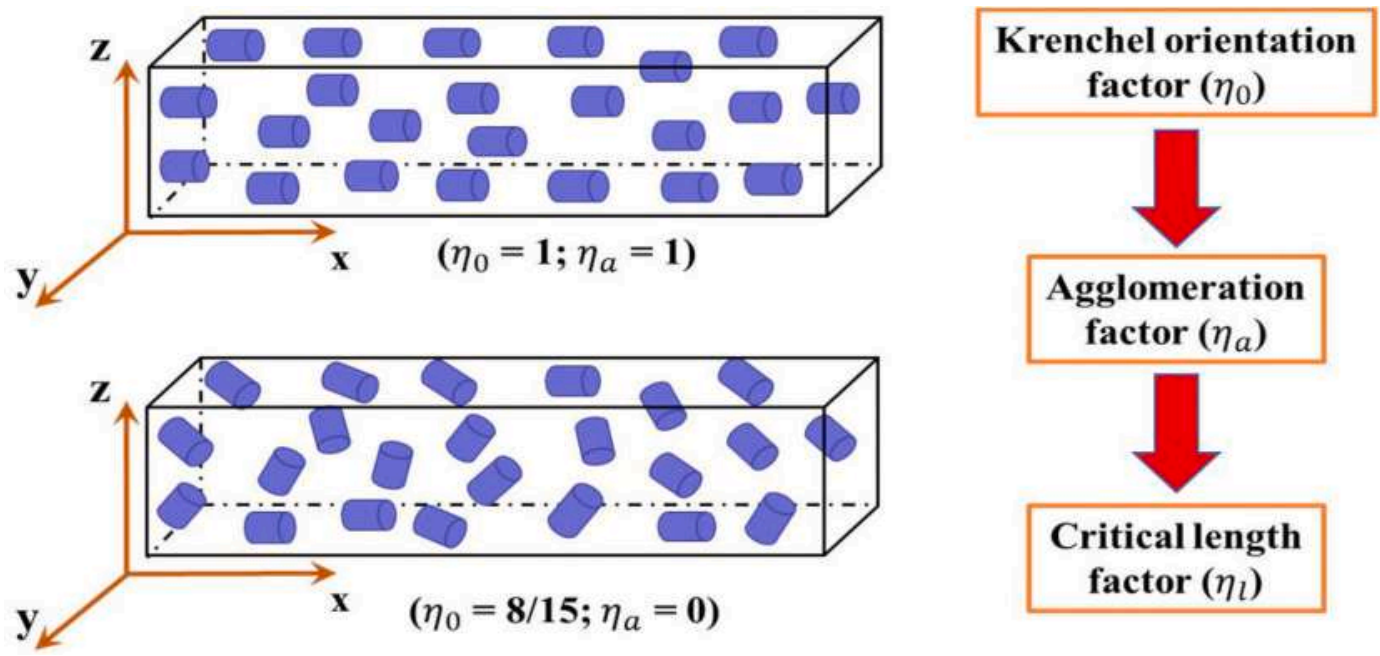

Fig. 1. Different orientation of graphene fibers in nanocomposite. 
two-phase composites are considered using two different combinations of two individual phases such as: (i) graphene/epoxy composite (GRC) and (ii) PZT/epoxy composites (PRC). From this, it is also clear that the graphene can be used as nanofillers/interphase. Smith and Auld (1991) predicted the effective properties of the piezoelectric composite made of PZT fibers incorporated in epoxy matrix. It is noticed that the effective properties estimated by them give better outcomes to control the thickness mode oscillations of thin composite plates. Therefore, in our present work, the micromechanical models are derived using the concept of single RVE (shows in Fig. 2a) to estimate the effective properties of the whole composite lamina. Fig. 2 (a) and (b) show an RVE of the composite lamina in which the piezoelectric (PZT) fibers are embedded in 1-direction. In the figure, it is also shown, an RVE is extracted from the composite lamina which represents the properties equivalent to that of its lamina because the strain energy stored in both is nearly equal.

To enhance the performance of composite, there should be an interaction between fiber and matrix. This could be done by treating the fiber or matrix or both before using them. The surface treatment of fiber is the most commonly used technique called sizing while such a treatment on the matrix to improve its adhesive property due to incorporation of nanofillers is promising and received great appreciation from the researchers (Tandon and Weng, 1984; Hashin, 1990; Wacker et al., 1998; Wan et al., 2005). In this paper, we are treating the epoxy matrix before embedding graphene nanofillers into it. For such a three-phase composite which is also known as a hybrid composite, the constitutive relation for the individual phases of GRPC can be expressed as:

$$
\left\{\sigma^{p}\right\}=\left[C^{p}\right]\left\{\epsilon^{p}\right\}-\left\{e^{p}\right\} E_{3}, \quad\left\{\sigma^{g}\right\}=\left[C^{g}\right]\left\{\epsilon^{g}\right\},\left\{\sigma^{m}\right\}=\left[C^{m}\right]\left\{\epsilon^{m}\right\}
$$

in which,

$$
\begin{aligned}
& \left\{\sigma^{r}\right\}=\left[\begin{array}{llllll}
\sigma_{1}^{r} & \sigma_{2}^{r} & \sigma_{3}^{r} & \sigma_{23}^{r} & \sigma_{13}^{r} & \sigma_{12}^{r}
\end{array}\right]^{T},\left\{\in^{r}\right\}=\left[\begin{array}{llllll}
\epsilon_{1}^{r} & \epsilon_{2}^{r} & \epsilon_{3}^{r} & \epsilon_{23}^{r} & \epsilon_{13}^{r} & \epsilon_{12}^{r}
\end{array}\right]^{T} \\
& \left\{e^{p}\right\}=\left[\begin{array}{llllll}
v_{p} e_{31}^{p} & e_{32}^{p} & e_{33}^{p} & 0 & 0 & 0
\end{array}\right]^{T} \\
& {\left[C^{r}\right]=\left[\begin{array}{llllll}
C_{11}^{r} & C_{12}^{r} & C_{13}^{r} & 0 & 0 & 0 \\
C_{12}^{r} & C_{22}^{r} & C_{23}^{r} & 0 & 0 & 0 \\
C_{13}^{r} & C_{23}^{r} & C_{33}^{r} & 0 & 0 & 0 \\
0 & 0 & 0 & C_{44}^{r} & 0 & 0 \\
0 & 0 & 0 & 0 & C_{55}^{r} & 0 \\
0 & 0 & 0 & 0 & 0 & C_{66}^{r}
\end{array}\right] \mathrm{r}=\mathrm{p}, \mathrm{g} \text { and } \mathrm{m}}
\end{aligned}
$$

The superscript $r$ is used to indicate the corresponding constituent phase and $p, g$ and $m$ superscripts represent the PZT fibers, graphene interphase and an epoxy matrix; where $\left\{\sigma^{r}\right\}$ and $\left\{\in^{r}\right\}$ denote the respective matrices for normal and shear stresses and strains; $\left[C^{r}\right]$ is matrix of the elastic stiffness coefficients of $r^{\text {th }}$ phase; and $\left\{e^{p}\right\}$ is the matrix for the piezoelectric coefficients of a PZT.

To satisfy perfect bond amongst PZT fiber, graphene nanofillers and the matrix, the uniform stress and strain conditions can be modelled as follows (Smith and Auld, 1991; Benveniste and Dvorak, 1992):

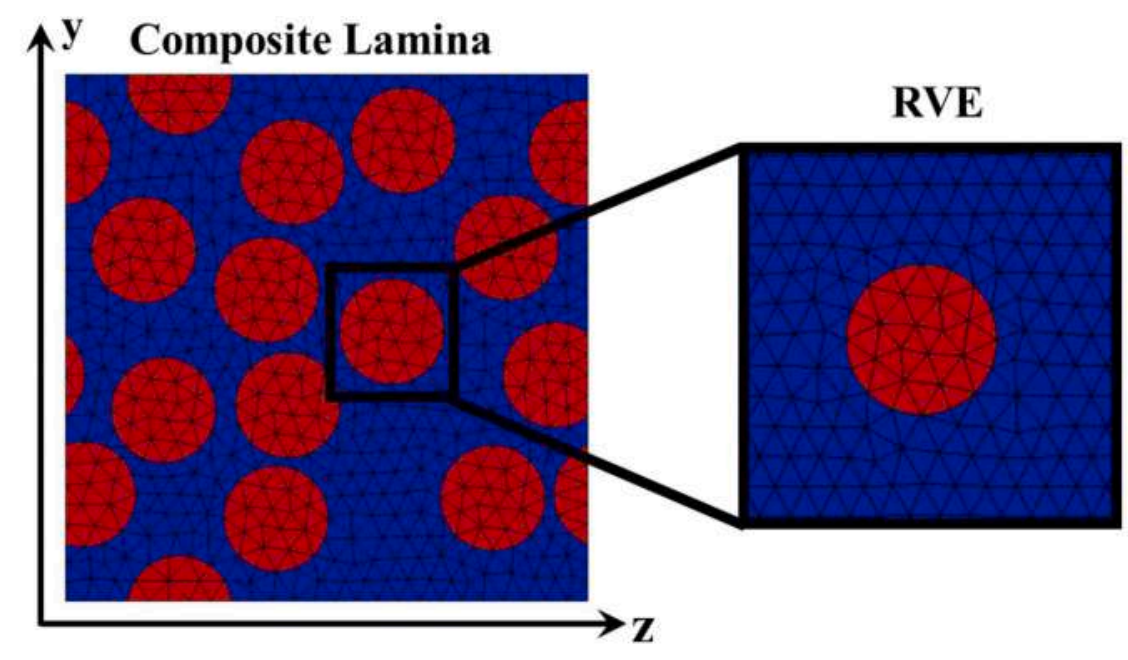

a

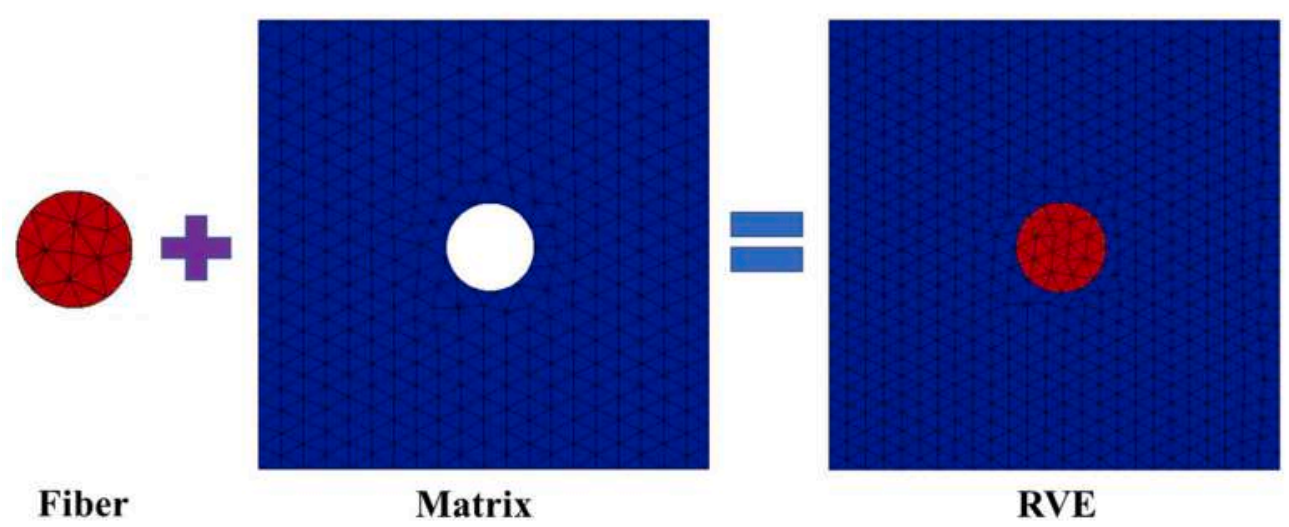

b

Fig. 2. (a): Schematic representation of single RVE with two-phase of composite lamina. (b): $2 \mathrm{D}$ schematic representation of single fiber RVE. 
$\left\{\begin{array}{l}\epsilon_{11}^{p} \\ \sigma_{22}^{p} \\ \sigma_{33}^{p} \\ \sigma_{23}^{p} \\ \sigma_{13}^{p} \\ \sigma_{12}^{p}\end{array}\right\}=\left\{\begin{array}{l}\epsilon_{11}^{g} \\ \sigma_{22}^{g} \\ \sigma_{33}^{g} \\ \sigma_{23}^{g} \\ \sigma_{13}^{g} \\ \sigma_{12}^{g}\end{array}\right\}=\left\{\begin{array}{l}\epsilon_{11}^{m} \\ \sigma_{22}^{m} \\ \sigma_{33}^{m} \\ \sigma_{23}^{m} \\ \sigma_{13}^{m} \\ \sigma_{12}^{m}\end{array}\right\}=\left\{\begin{array}{l}\epsilon_{11}^{c} \\ \sigma_{22}^{c} \\ \sigma_{33}^{c} \\ \sigma_{23}^{c} \\ \sigma_{13}^{c} \\ \sigma_{12}^{c}\end{array}\right\}$

The uniform stress and strain condition expressed in above Eq. (10) signifies that the strains in all respective phases of composite along the fiber direction are similar and stresses in these same phases are same along the direction normal to the fiber direction.

Using simple ROM condition, we can obtain following equation:

$v_{p}\left\{\begin{array}{l}\sigma_{11}^{p} \\ \epsilon_{22}^{p} \\ \epsilon_{33}^{p} \\ \epsilon_{23}^{p} \\ \epsilon_{13}^{p} \\ \epsilon_{12}^{p}\end{array}\right\}+v_{g}\left\{\begin{array}{l}\sigma_{11}^{g} \\ \epsilon_{22}^{g} \\ \epsilon_{33}^{g} \\ \epsilon_{23}^{g} \\ \epsilon_{13}^{g} \\ \epsilon_{12}^{g}\end{array}\right\}+v_{m}\left\{\begin{array}{l}\sigma_{11}^{m} \\ \epsilon_{22}^{m} \\ \epsilon_{33}^{m} \\ \epsilon_{23}^{m} \\ \epsilon_{13}^{m} \\ \epsilon_{12}^{m}\end{array}\right\}=\left\{\begin{array}{c}\sigma_{11}^{c} \\ \epsilon_{22}^{c} \\ \epsilon_{33}^{c} \\ \epsilon_{23}^{c} \\ \epsilon_{13}^{c} \\ \epsilon_{12}^{c}\end{array}\right\}$

where, the superscript $C$ indicates the quantities of composite, whereas $v_{p}, v_{g}$ and $v_{m}$ are the volume fractions of a PZT fiber, graphene interphase and the epoxy matrix, respectively. For two-phase model, we can use condition of volume fraction such as: $v_{p}+v_{m}=1$ while for threephase model, it can be written as: $v_{p}+v_{g}+v_{m}=1$.

As the thickness of the graphene filler is too small, it can be closely packed to make spacing amongst the fiber and matrix. Also, the assumption of same longitudinal strain in 1- or in fiber direction in all respective phases is not strictly effective unless the bottom and top surfaces are bonded to rigid membranes and are uniformly pressed in the thickness direction. However, the assumption gives better estimates for applications of beams and plates as actuators for uniformly distributed PZTs and graphene with very small gap among them.

From Eqs. (9-11), the following stress and strain vectors of homogenized GRPC can be written in terms of the corresponding stress and strain vectors of fiber, interphase and matrix phases:

$\left\{\sigma^{c}\right\}=\left[C_{1}\right]\left\{\in^{p}\right\}+\left[C_{2}\right]\left\{\in^{g}\right\}+\left[C_{3}\right]\left\{\epsilon^{m}\right\}-\left\{e_{1}\right\} E_{3}$

$\left[C_{4}\right]\left\{\in^{p}\right\}-\left[C_{5}\right]\left\{\in^{g}\right\}=\left\{e_{2}\right\} E_{3}$

$\left\{\in^{c}\right\}=\left[V_{1}\right]\left\{\in^{p}\right\}+\left[V_{2}\right]\left\{\in^{g}\right\}+\left[V_{3}\right]\left\{\in^{m}\right\}$ and

$\left[C_{5}\right]\left\{\epsilon^{g}\right\}-\left[C_{6}\right]\left\{\epsilon^{m}\right\}=0$

where,

$\begin{aligned} {\left[C_{1}\right] } & =\left[\begin{array}{llllll}v_{p} C_{11}^{p} & v_{p} C_{12}^{p} & v_{p} C_{13}^{p} & 0 & 0 & 0 \\ C_{12}^{p} & C_{22}^{p} & C_{23}^{p} & 0 & 0 & 0 \\ C_{13}^{p} & C_{23}^{p} & C_{33}^{p} & 0 & 0 & 0 \\ 0 & 0 & 0 & C_{44}^{p} & 0 & 0 \\ 0 & 0 & 0 & 0 & C_{55}^{p} & 0 \\ 0 & 0 & 0 & 0 & 0 & C_{66}^{p}\end{array}\right],\left[C_{2}\right] \\ & =\left[\begin{array}{llllll}v_{g} C_{11}^{g} & v_{g} C_{12}^{g} & v_{g} C_{13}^{g} & 0 & 0 & 0 \\ 0 & 0 & 0 & 0 & 0 & 0 \\ 0 & 0 & 0 & 0 & 0 & 0 \\ 0 & 0 & 0 & 0 & 0 & 0 \\ 0 & 0 & 0 & 0 & 0 & 0 \\ 0 & 0 & 0 & 0 & 0 & 0\end{array}\right]\end{aligned}$
$\left[C_{3}\right]=\left[\begin{array}{llllll}v_{m} C_{11}^{m} & v_{m} C_{12}^{m} & v_{m} C_{13}^{m} & 0 & 0 & 0 \\ 0 & 0 & 0 & 0 & 0 & 0 \\ 0 & 0 & 0 & 0 & 0 & 0 \\ 0 & 0 & 0 & 0 & 0 & 0 \\ 0 & 0 & 0 & 0 & 0 & 0 \\ 0 & 0 & 0 & 0 & 0 & 0\end{array}\right],\left[C_{4}\right]=\left[\begin{array}{llllll}1 & 0 & 0 & 0 & 0 & 0 \\ C_{12}^{p} & C_{22}^{p} & C_{23}^{p} & 0 & 0 & 0 \\ C_{13}^{p} & C_{23}^{p} & C_{33}^{p} & 0 & 0 & 0 \\ 0 & 0 & 0 & C_{44}^{p} & 0 & 0 \\ 0 & 0 & 0 & 0 & C_{55}^{p} & 0 \\ 0 & 0 & 0 & 0 & 0 & C_{66}^{p}\end{array}\right]$

$\left[C_{5}\right]=\left[\begin{array}{llllll}1 & 0 & 0 & 0 & 0 & 0 \\ C_{12}^{g} & C_{22}^{g} & C_{23}^{g} & 0 & 0 & 0 \\ C_{13}^{g} & C_{23}^{g} & C_{33}^{g} & 0 & 0 & 0 \\ 0 & 0 & 0 & C_{44}^{g} & 0 & 0 \\ 0 & 0 & 0 & 0 & C_{55}^{g} & 0 \\ 0 & 0 & 0 & 0 & 0 & C_{66}^{g}\end{array}\right],\left[C_{6}\right]=\left[\begin{array}{llllll}1 & 0 & 0 & 0 & 0 & 0 \\ C_{12}^{m} & C_{22}^{m} & C_{23}^{m} & 0 & 0 & 0 \\ C_{13}^{m} & C_{23}^{m} & C_{33}^{m} & 0 & 0 & 0 \\ 0 & 0 & 0 & C_{44}^{m} & 0 & 0 \\ 0 & 0 & 0 & 0 & C_{55}^{m} & 0 \\ 0 & 0 & 0 & 0 & 0 & C_{66}^{m}\end{array}\right]$

$\left\{e_{1}\right\}=\left\{\begin{array}{llllll}v_{p} e_{31}^{p} & e_{32}^{p} & e_{33}^{p} & 0 & 0 & 0\end{array}\right\}^{T}, \quad\left\{e_{2}\right\}=\left\{\begin{array}{llllll}0 & e_{32}^{p} & e_{33}^{p} & 0 & 0 & 0\end{array}\right\}^{T}$

$\left[V_{1}\right]=\left[\begin{array}{llllll}1 & 0 & 0 & 0 & 0 & 0 \\ 0 & v_{p} & 0 & 0 & 0 & 0 \\ 0 & 0 & v_{p} & 0 & 0 & 0 \\ 0 & 0 & 0 & v_{p} & 0 & 0 \\ 0 & 0 & 0 & 0 & v_{p} & 0 \\ 0 & 0 & 0 & 0 & 0 & v_{p}\end{array}\right],\left[V_{2}\right]=\left[\begin{array}{cccccc}0 & 0 & 0 & 0 & 0 & 0 \\ 0 & v_{g} & 0 & 0 & 0 & 0 \\ 0 & 0 & v_{g} & 0 & 0 & 0 \\ 0 & 0 & 0 & v_{g} & 0 & 0 \\ 0 & 0 & 0 & 0 & v_{g} & 0 \\ 0 & 0 & 0 & 0 & 0 & v_{g}\end{array}\right]$ and,

$\left[V_{3}\right]=\left[\begin{array}{llllll}0 & 0 & 0 & 0 & 0 & 0 \\ 0 & v_{m} & 0 & 0 & 0 & 0 \\ 0 & 0 & v_{m} & 0 & 0 & 0 \\ 0 & 0 & 0 & v_{m} & 0 & 0 \\ 0 & 0 & 0 & 0 & v_{m} & 0 \\ 0 & 0 & 0 & 0 & 0 & v_{m}\end{array}\right]$

By substituting Eq. (9) into Eq. (12), a following constitutive equation for the GRPC lamina is given as:

$\left\{\sigma^{c}\right\}=\left[C^{c}\right]\left\{\epsilon^{c}\right\}-\left\{e^{c}\right\} E_{3}$

where $\left[C^{c}\right]$ and $\left\{e^{c}\right\}$ are the effective elastic and piezoelectric matrices of GRPC, respectively, and can be written as following:

$\left[C^{c}\right]=\left[C_{1}\right]\left[V_{5}\right]^{-1}+\left[C_{7}\right]\left[V_{6}\right]^{-1}$
$\left\{e^{c}\right\}=\left\{e_{1}\right\}+\left(\left[C_{1}\right]\left[V_{5}\right]^{-1}\left[V_{4}\right]\left[C_{6}\right]^{-1}+\left[C_{7}\right]\left[V_{6}\right]^{-1}\left[V_{1}\right]\left[C_{4}\right]^{-1}\right)\left\{e_{2}\right\}$

where,

$$
\begin{aligned}
& {\left[C_{7}\right]=\left[C_{3}\right]+\left[C_{2}\right]\left[C_{5}\right]^{-1}\left[C_{6}\right],\left[V_{4}\right]=\left[V_{3}\right]+\left[V_{2}\right]\left[C_{5}\right]^{-1}\left[C_{6}\right]} \\
& {\left[V_{5}\right]=\left[V_{1}\right]+\left[V_{4}\right]\left[C_{6}\right]^{-1}\left[C_{4}\right], \quad\left[V_{6}\right]=\left[V_{4}\right]+\left[V_{1}\right]\left[C_{4}\right]^{-1}\left[C_{6}\right] \text {, and }} \\
& \left\{e^{c}\right\}=\left\{\begin{array}{llllll}
e_{31}^{c} & e_{32}^{c} & e_{33}^{c} & 0 & 0 & 0
\end{array}\right\}^{T}
\end{aligned}
$$

\section{Finite element model}

Computational methods such as finite element models (FEM) have become more user friendly and popular as these models offer several advantages as compared to the analytical models. To get realistic microstructures, the complicated geometry and electro-thermomechanical properties of the individual phases can be easily introduced. Therefore, to define and solve the problem of micromechanics of GRC using a computational approach, it is significant to identify the micromechanical model and analysis. Fig. 3 illustrates the detailed framework of work which will be carried out to achieve the effective properties of composite. The FE models are carried out to validate the assumptions made in micromechanical modelling such as uniform strain in composite and its constituents along the fiber direction and uniform 
stress in composite and its constituents normal to the fiber direction (shown in Fig. 4). In this FE analysis, we considered the different types of RVE considering different numbers and shapes of fiber, as shown in Fig. 4(a-c).

For inclusion type of fibers, the cylindrical and ellipsoidal shapes are considered. We can model the RVE using different types of geometry consideration or array packing such as square and hexagonal packing. In case of a circular cross-section of fibers, the maximum fiber volume fraction for RVE of the square array of packing is 0.786 , while it is 0.907 in hexagonal array of packing. Nevertheless, these are ideal circumstances only used for theoretical analysis. In actual, there can be variation not only due to fiber diameter but also because of the irregular packing, due to this, it is critical to attain a volume fraction greater than 0.7 . Hence, we considered up to 0.4 volume fraction of PZT fiber for MOM modelling. In this FE analysis, we assumed both types of RVEs (i. e., square as well as hexagonal RVE).

Despite the fact that the elastic modulus of any material is the slope of its stress-strain curve in the elastic region and hence, on the application of small displacement to the RVE (various loading and boundary conditions), using the slope of stress-strain, the effective elastic con- stants can be estimated for any composite which obeys Hooke's law. Firstly, we considered that $\mathrm{x}, \mathrm{y}$ and $\mathrm{z}$ indicate the coordinates corresponding to 1, 2 and 3-axes of RVE, respectively. The advantages of FE models are that one can obtain the three effective constants with the application of single boundary and loading conditions, as shown in Fig. 5 (a (i) and b (i)). For example, in order to compute the effective elastic constant $C_{22}$ of PRC or GRPC, the RVE shown in Fig. 5a (i and ii) can be deformed in such a way that the normal strain is only exist in 2or y-direction while remaining strain components are zero. Similarly, the electric fields at all surfaces are set to zero in case of piezoelectric composite. In order to attain such states of strain, a uniform normal displacement at the five surfaces of RVE need to set to zero as shown in Fig. 5a (i and ii). A uniform normal displacement along the 2-direction needs to be applied on the surface of the RVE such that it is subjected to average strain in y-direction only. Similar boundary and loading conditions can be applied for obtaining the remaining elastic coefficients such as $C_{11}, C_{12}, C_{13}, C_{23}, C_{33}, C_{44}$, and $C_{66}$. The detailed steps required for the determination of these coefficients are described elsewhere (Kundalwal et al., 2019, 2020a, 2020b; Shingare and Kundalwal, 2019a; Shingare and Kundalwal, 2020).

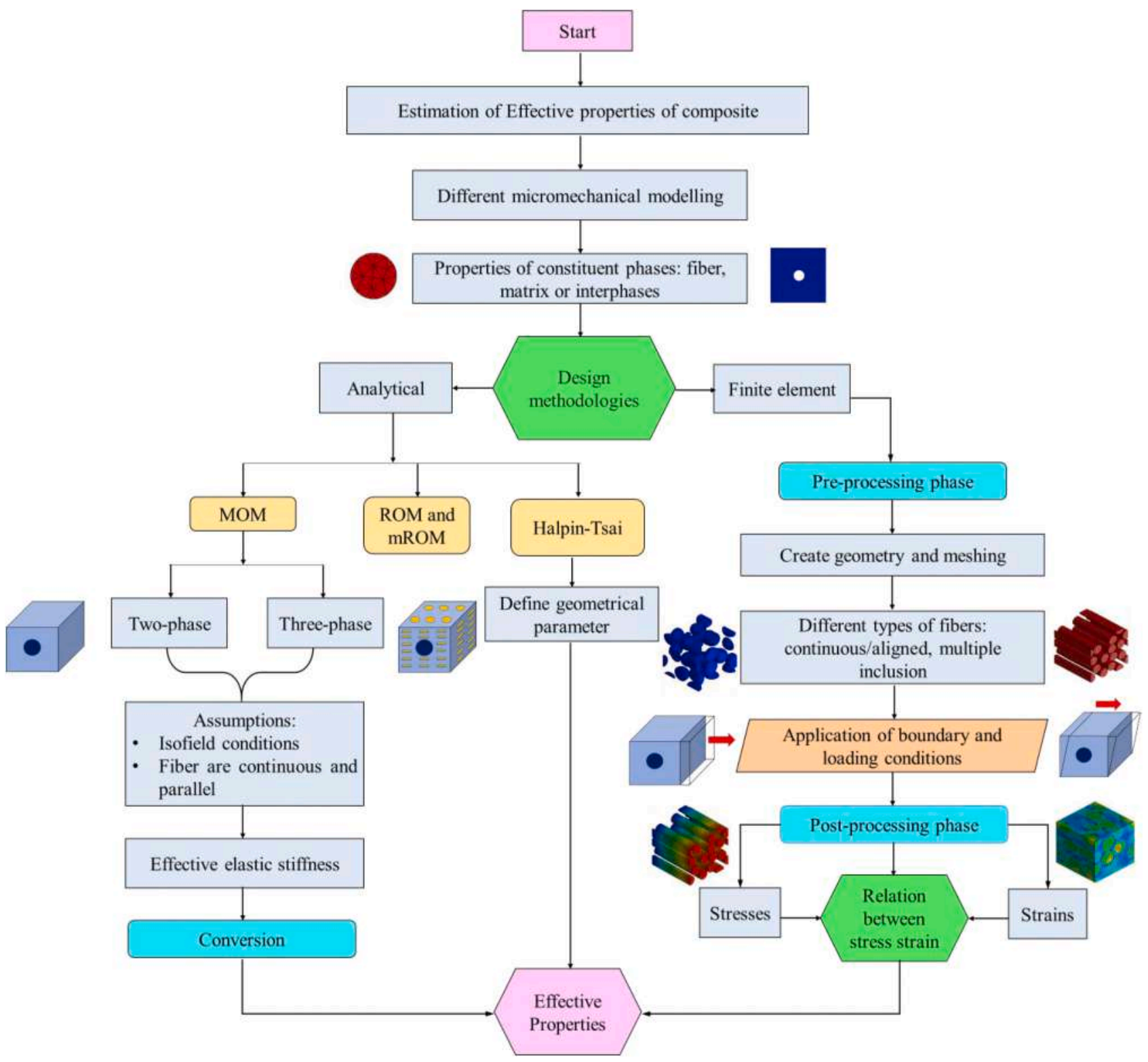

Fig. 3. The detailed flowchart of micromechanical analysis of composite using different modelling techniques. 


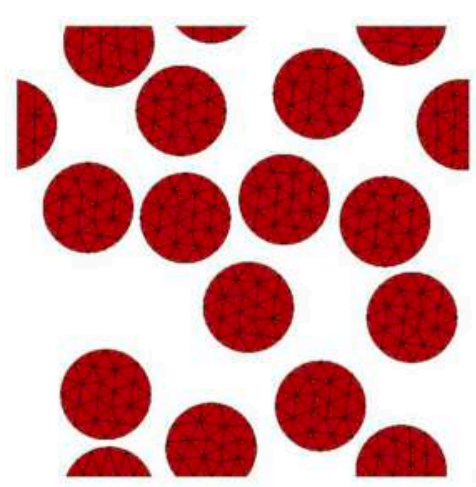

Fiber
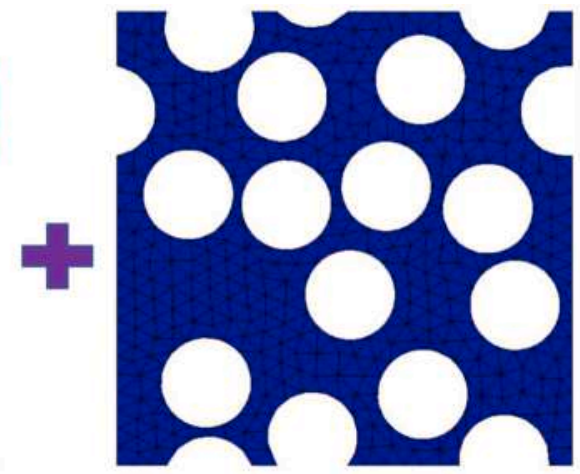

Matrix

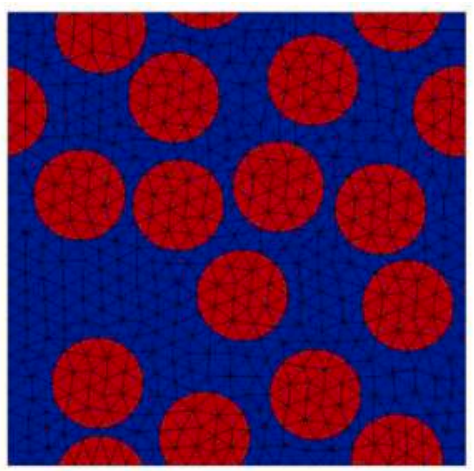

RVE

(a): 2D schematic representation of multiple fiber distribution in RVE.

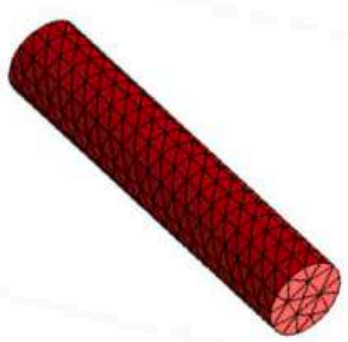

Fiber
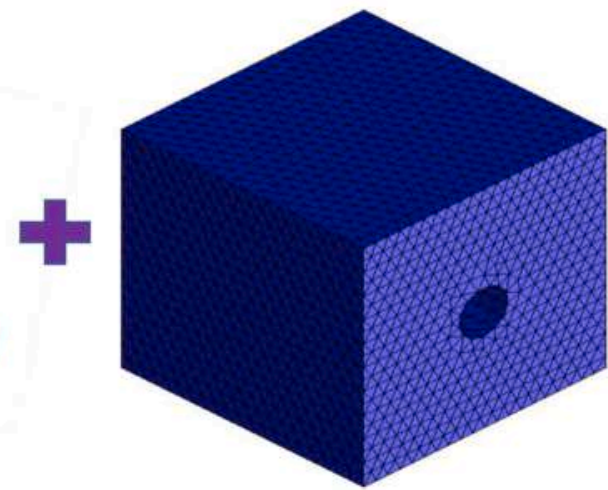

Matrix

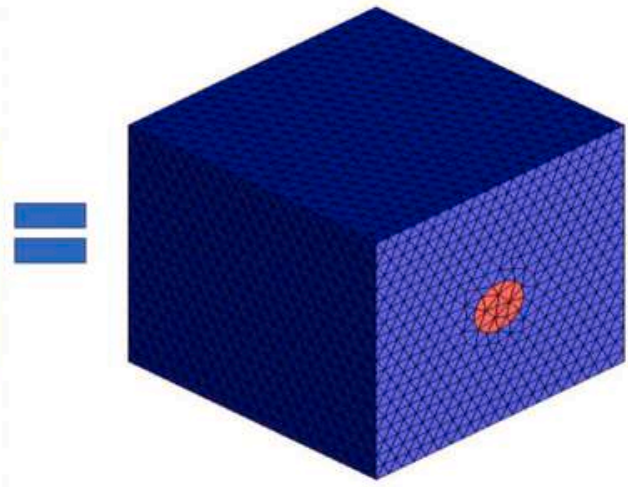

RVE

(b): 3D schematic representation of single fiber RVE.

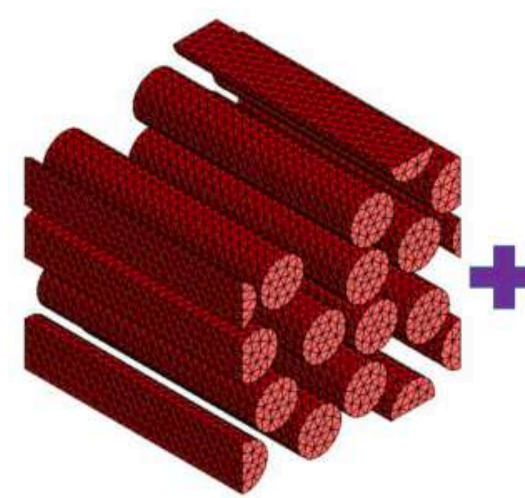

Fiber

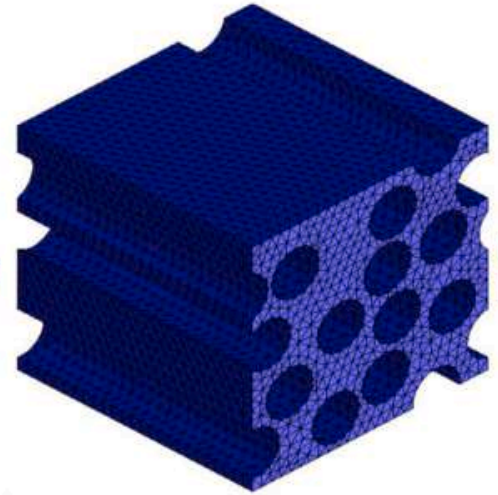

Matrix

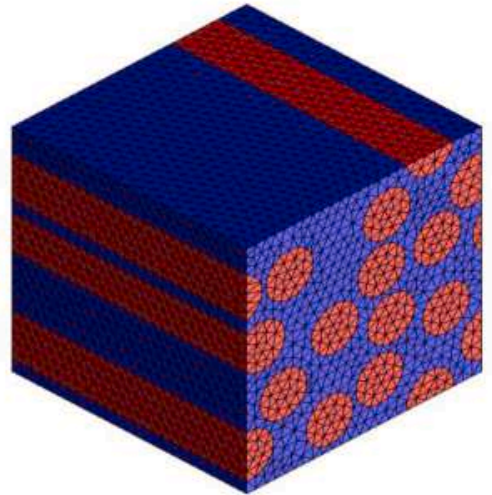

RVE

(c): 3D schematic representation of multiple fiber distribution in RVE.

Fig. 4. (a): 2Dschematic representation of multiple fiber distribution in RVE. (b): 3Dschematic representation of single fiber RVE. (c): 3Dschematic representation of multiple fiber distribution in RVE. 
As we know, every FE analysis is divided into pre-processing, computation and post-processing steps. The Digimat analysis (E-xstream.com, 2018) is not restricted to the definition of material laws but necessitates the description of the microstructure morphology of the composite and the loading conditions. For instance, RVE is modelled using graphene as inclusion phase and epoxy matrix. Such an inclusion type graphene particles are considered as uniformly as well as randomly distributed in epoxy matrix by predefining volume fraction, shape parameters (cylindrical, ellipsoidal, prism, platelet, etc.), number of inclusions, aspect ratio and diameter of inclusion. To represent the microstructure of different volume fraction of composites, the 3D RVEs (shown in Fig. 4 (b and c)) of composites lamina are carried out using FE software that uses the user-defined volume fraction method for particle distribution. The randomness and orientation of inclusion particles in the RVE are defined by sequential orientation with required angle/random option. In our work, the overall process from the pre-processor to the post-processor stage is presented. For such an analysis to be completely well-defined, proper loadings must be imposed on the RVE. For all FE code interfaces based on the in-built mesher, it also permits starting and checking the progress of the FE simulation. To view the 3D detailed results, the default FE solver can be used, and we can carry-out cut section analysis into 3D model and different contour plots can be visualized in post-processor section. Fig. 6(a-e) depict the distribution of displacement, stresses, strains considering deformation scale factor in $\mathrm{x}$-direction and contact status of fiber inclusion and matrix in RVE. Fig. 6 (a and b) show the distribution of displacement along with their

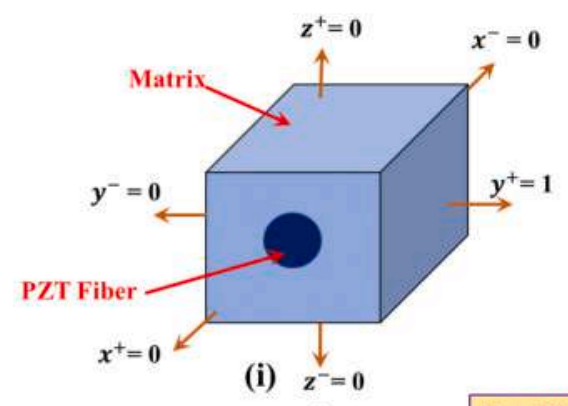

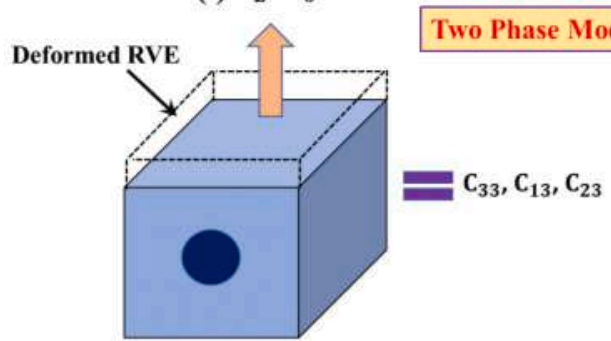

(iii)

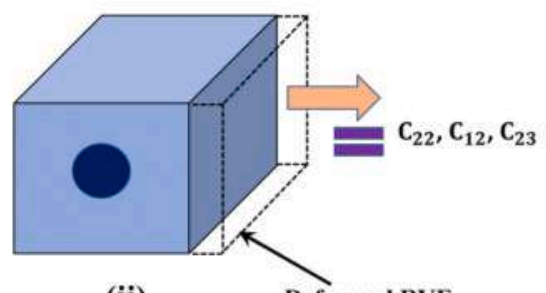

(ii)

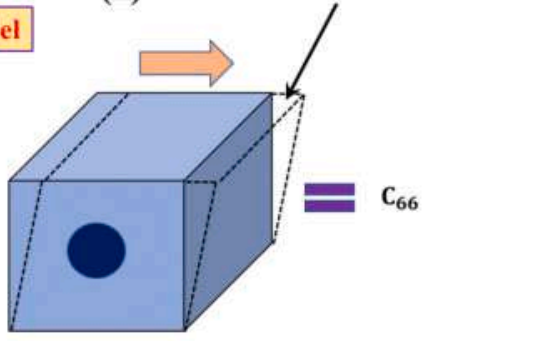

(iv)

(a): Two-phase PRC RVEs under different types of loadings and boundary conditions.

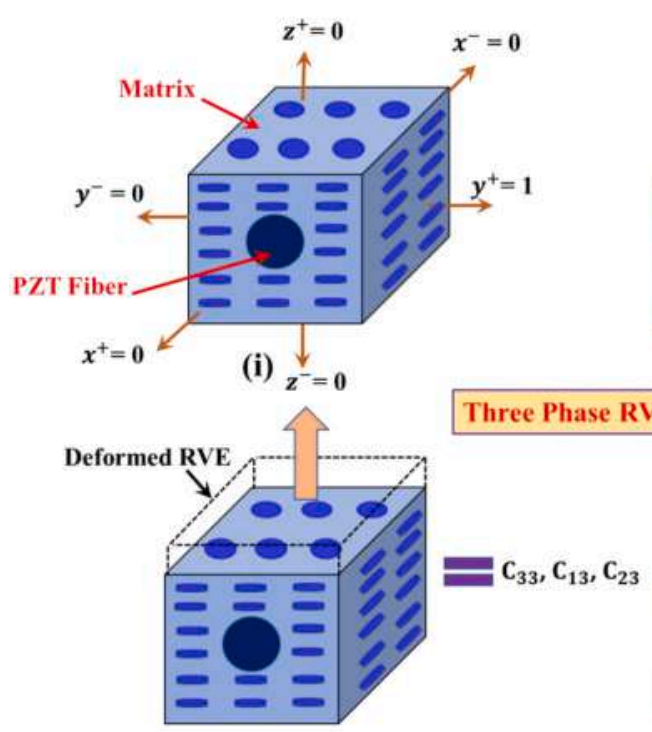

(iii)

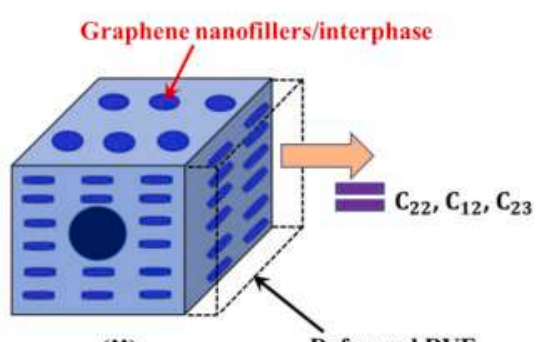

(ii)

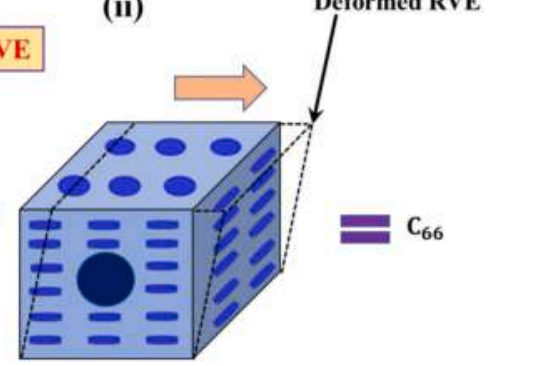

(iv)

(b): Three-phase GRPC RVEs under different types of loadings and boundary conditions.

Fig. 5. (a): Two-phase PRC RVEs under different types of loadings and boundary conditions. (b): Three-phase GRPC RVEs under different types of loadings and boundary conditions. 
cut-section in the GRC RVE when subjected to default boundary and loading conditions. The stresses in the GRC RVE (as shown in Fig. 6 (c)) reveal that the perfect bond exists between the graphene nanofillers and epoxy matrix due to the high strength and high modulus of graphene as that of the matrix. The load applied to the RVE is mainly exerted on the matrix and then transferred or distributed to the graphene nanofillers. It results in the stresses and strains induced in the GRC RVE which are showed in Fig. 6 (c and d). To identify the defects occurred, the study on the state of stresses and strains will be very beneficial. The important causes of failure of nanocomposites during their fabrication and applications may be because of the initiated defects and its propagation when subjected to applied stresses. The distribution of total strains in the GRC RVE in x-direction (Fig. 6 (d)) undoubtedly shows that the strain in the matrix is greater than that of the graphene nanofillers. It is attributed to fact that the embedded graphene nanofillers have high Young's modulus as compared to epoxy matrix. Fig. 6 (e) illustrates the contact status between the graphene reinforcement and epoxy matrix.

\section{Result and discussion}

In order to illustrate the performance of effective properties of GRC using different models such as rules-of-mixture (ROM), modified rulesof-mixture (mROM), Halpin-Tsai (HT), two- and three-phase mechanics of material (MOM) and finite element (FE) models presented in previous sections, the numerical results are presented in this section. The material properties of constituents of GRC enlisted in Table 2. The geometrical parameters used for graphene platelets (GPLs): width $w=$ $1.5 \mu \mathrm{m}$, thickness $t=1.5 \mathrm{~nm}$ and length $l=2.5 \mu \mathrm{m}$; the GPL weight fraction of 0.1 wt.\% is exactly equal to 0.112 vol.\% (Rafiee et al., 2009). For the sake of brevity, we mentioned graphene for its derivatives (a)
(c)
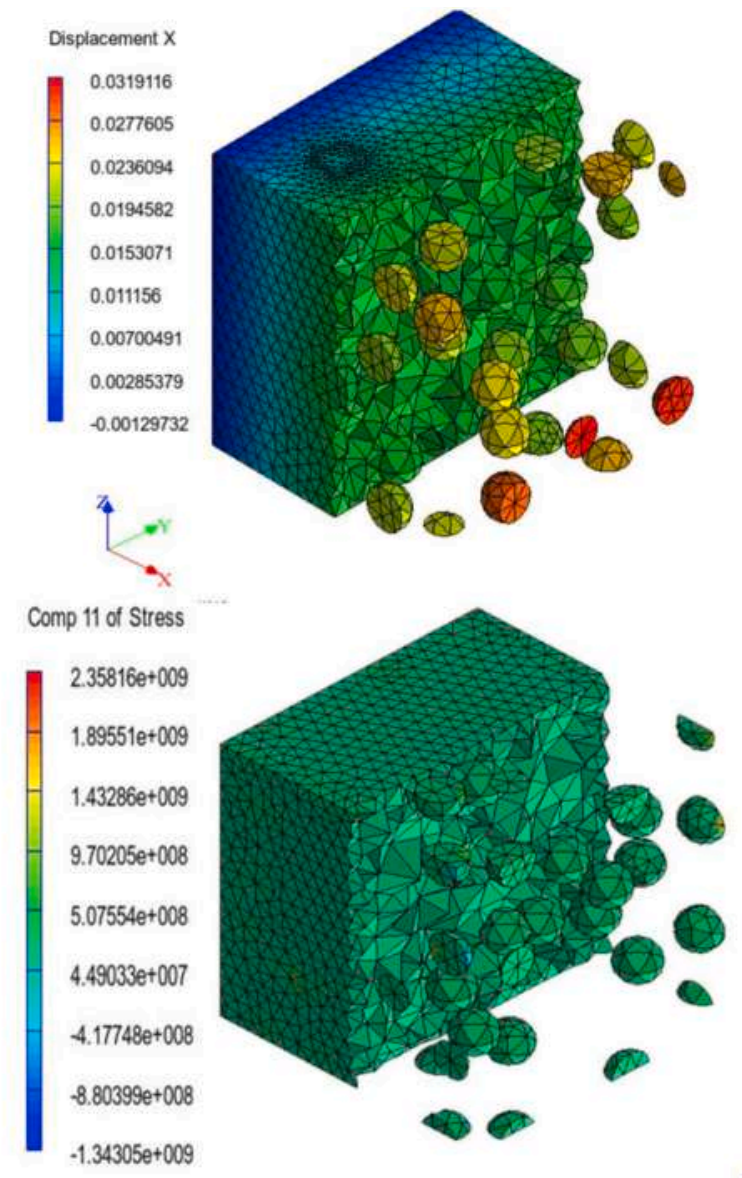

$$
\begin{array}{r}
\text { Comp } 11 \text { of Stress } \\
2.35816 e+009 \\
1.89551 e+009 \\
1.43286 e+009 \\
9.70205 e+008 \\
5.07554 e+008 \\
4.49033 e+007 \\
4.17748 e+008 \\
-8.80399 e+008 \\
-1.34305 e+009
\end{array}
$$

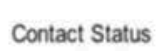

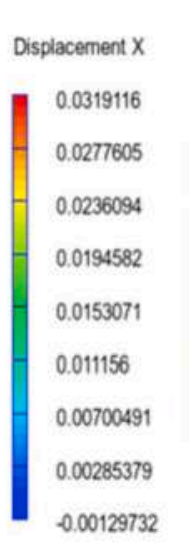

Comp 11 of Total Strain

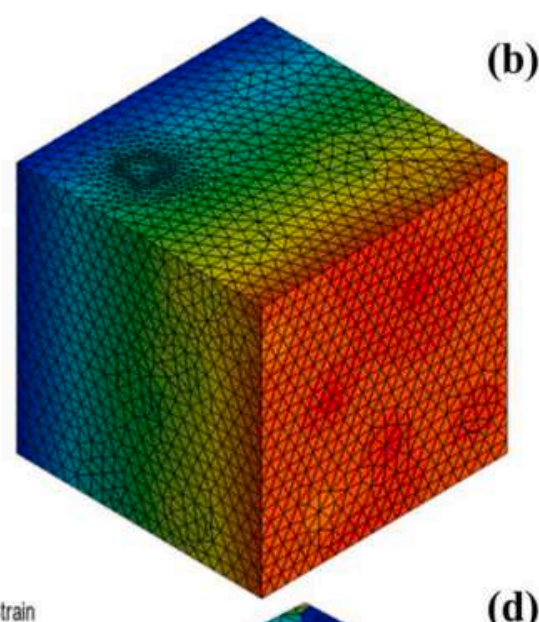

(d)

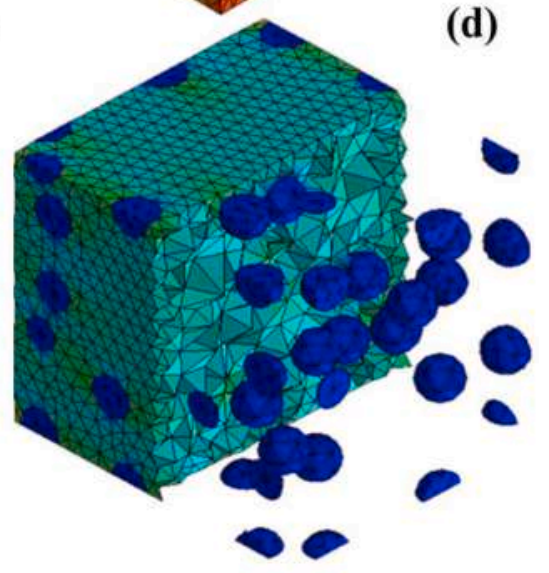

(e)

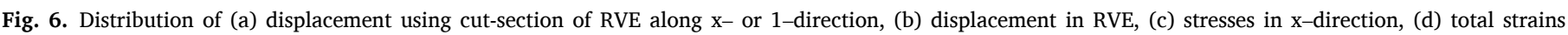
considering deformation scale factor in $\mathrm{x}$-direction and (e) contact status between the graphene and epoxy in GRC RVE. 
such as graphene platelets.

Firstly, using different ROM methods, the effective elastic modulus of GRC is determined for $1 \%$ volume fraction of graphene $\left(v_{g}\right)$, summarized in Table 3. From Table 3, it can be clearly observed that the orientation, agglomeration and critical length factors play a significant role while estimating the effective elastic modulus of composite. We have also determined the effective modulus of composite by taking some random values of Krenchel orientation factors to understand how it effects on effective properties. The results obtained from the different ROM methods are found to be in excellent coherence with results predicted using the MOM and FE models which are tabulated in Table 6. In Table $3, P_{i} \cos \theta$ indicates the Legendre polynomial of $i^{\text {th }}$ degree and $<$ $\left.P_{i} \cos \theta\right\rangle$ denotes the average value. $\left\langle P_{2} \cos \theta\right\rangle$ is Herman's orientation factor, while for the higher orientation of graphene in a composite, the values of $\left\langle P_{2} \cos \theta\right\rangle$ and $\left\langle P_{4} \cos \theta\right\rangle$ should be higher.

In $\mathrm{FE}$ modelling, for finding the reliable results, the mesh convergence was carried out using conforming tetra mesh type and which are shown in Table 4. The FE mesh convergence demonstrates how many numbers of nodes and elements are necessary to ensure that the outcomes of the analysis are not influenced by changing the size of nodes and elements. Table 4 shows that the value of elastic modulus does not change after certain number of node and elements, and we carried out such convergence studies for determining the modulus of composite.

Using different methods such as MOM and FE model, the effective elastic Young's and shear moduli of GRC reinforced with continuous/ aligned graphene nanofillers are determined for $v_{g}=0.05-10 \%$, summarized in Tables 5 and 6. In this MOM model, we used the two phases such as graphene and epoxy matrix. The FE modelling is carried out to validate the hypothesis made in analytical modelling such as uniform strain in composite and its constituents along the fiber direction and uniform stress in composite and its constituents normal to the fiber direction. From Tables 5 and 6 , it can be observed that longitudinal Young's modulus of GRC gives near about $\sim 100 \%$ agreement between the MOM and FE model while transverse Young's and shear modulus are shown the marginal difference between both models. It is attributed to fact that longitudinal modulus follows perfect uniform stresses/strains condition while these transverse and shear modulus depends on matrix properties and also influenced by in-plane behavior of composite. Especially in case of volume fraction 0.05 and 0.1 , the elastic modulus of composite matches with the results reported in previous literature using experimental investigations (Rafiee et al., 2010; Zaman et al., 2011, 2012; Tang et al., 2013) presented in Table 8.

The effective elastic moduli of GRC are determined for $0.1-0.5 \%$ volume fraction of graphene by using different methods such as ROM, mROM, two-phase MOM, FE model and HT method considering geometrical parameters for graphene, as summarized in Table 7 . Moreover, in case of ROM, the Krenchel orientation, critical length and agglomeration factor plays a vital role.

The presented results using FE model are validated with the earlier reported experimental estimates, as shown in Table 8 for GRC. From this, it can be seen that all predicted results using present FE models are found to be in better agreement with experimental predictions for

Table 2

Material properties of constituents of GRPC.

\begin{tabular}{|c|c|c|c|}
\hline Properties & GPLs & Epoxy & PZT 5H \\
\hline $\begin{array}{l}\text { Elastic constants } \\
\quad \text { (GPa) }\end{array}$ & 1010 & 2.85 & $\begin{array}{l}C_{11}=151, C_{12}=98, C_{13}=96 \\
C_{33}=124, C_{44}=C_{66}=23\end{array}$ \\
\hline Poisson's ratio & 0.186 & 0.3 & - \\
\hline Density $\left(\mathrm{kg} / \mathrm{m}^{3}\right)$ & 1060 & 1180 & - \\
\hline $\begin{array}{l}\text { Piezoelectric } \\
\text { constants (C/ } \\
\mathrm{m}^{2} \text { ) }\end{array}$ & - & - & $\begin{array}{l}e_{31}=-5.1, e_{32}=-5.1, e_{33}= \\
27\end{array}$ \\
\hline Ref. & $\begin{array}{l}\text { Rafiee } \\
\text { et al. } \\
(2009)\end{array}$ & $\begin{array}{l}\text { Rafiee } \\
\text { et al. } \\
\text { (2009) }\end{array}$ & Kumar and Chakraborty (2009) \\
\hline
\end{tabular}

Table 3

The effective elastic modulus of GRC using different ROM $\left(v_{g}=1 \%\right)$.

\begin{tabular}{|c|c|c|}
\hline Analytical model & $\begin{array}{l}\text { Elastic } \\
\text { modulus } E_{c} \\
(\mathrm{GPa})\end{array}$ & $\begin{array}{l}\text { Orientation, alignment and } \\
\text { agglomeration factors }\end{array}$ \\
\hline $\begin{array}{l}\text { Rules-of-mixture (ROM) } \\
\quad E_{c}=E_{f} v_{f}+E_{m} v_{m}\end{array}$ & 12.921 & - \\
\hline $\begin{array}{l}\text { Modified rules-of-mixture } \\
\quad(\mathrm{mROM}) \\
E_{c}=\eta_{0} \eta_{l} \eta_{a} E_{f} v_{f}+E_{m} v_{m}\end{array}$ & 12.921 & $\begin{array}{l}\eta_{1}=1, \quad \eta_{0}=1 \text { (Perfect } \\
\text { aligned and 2D random } \\
\text { fiber) }\end{array}$ \\
\hline $\begin{array}{l}\eta_{0}=\frac{8}{15}+\frac{8}{21}\left\langle P_{2} \cos \theta\right\rangle+\frac{3}{35}\langle \\
\left.P_{4} \cos \theta\right\rangle \\
\text { where }\end{array}$ & 8.204 & $\begin{array}{l}\eta_{1}=1, \eta_{0}= \\
0.533(3 \text { Dreinforced random } \\
\text { fiber) }\end{array}$ \\
\hline $\begin{array}{l}\text { i. }\left\langle P_{2} \cos \theta\right\rangle=\left\langle P_{4} \cos \theta\right\rangle=1 \\
\eta_{0}=1\end{array}$ & 12.921 & $\begin{array}{l}\eta_{1}=1, \quad \eta_{0}=1(\text { low } \\
\left.\text { agglomeration } \eta_{a}=1\right)\end{array}$ \\
\hline $\begin{array}{l}\text { (for perfectly oriented) } \\
\text { ii. }\left\langle P_{2} \cos \theta\right\rangle=\left\langle P_{4} \cos \theta\right\rangle=0\end{array}$ & 2.821 & $\begin{array}{l}\eta_{1}=1, \quad \eta_{0}=1 \text { (high } \\
\left.\text { agglomeration } \eta_{a}=0\right)\end{array}$ \\
\hline \multirow[t]{4}{*}{$\begin{array}{l}\eta_{0}=\frac{8}{15} \\
\text { (for } 3 \mathrm{D} \text { randomly oriented) }\end{array}$} & 8.204 & $\begin{array}{l}\eta_{0}=0.533, \quad \eta_{1}=1, \quad \eta_{a}= \\
1(3 \mathrm{D} \text { randomly oriented } \\
\text { fiber })\end{array}$ \\
\hline & 2.821 & $\begin{array}{l}\eta_{0}=0.533, \quad \eta_{1}=1, \quad \eta_{a}= \\
0(3 \mathrm{D} \text { randomly oriented } \\
\text { fiber })\end{array}$ \\
\hline & 9.891 & $\begin{array}{l}\eta_{0}=0.70, \quad \eta_{1}=1, \quad \eta_{a}= \\
1(3 \mathrm{D} \text { randomly oriented } \\
\text { fiber })\end{array}$ \\
\hline & 10.396 & $\begin{array}{l}\eta_{0}=0.75, \quad \eta_{1}=1, \quad \eta_{a}= \\
1(3 \mathrm{D} \text { randomly oriented } \\
\text { fiber })\end{array}$ \\
\hline $\begin{array}{l}\text { Mechanics of materials (MOM) } \\
\text { Model }\end{array}$ & 12.21 & $\begin{array}{l}\text { (Perfect bonding and 2D, } \\
\text { 3D aligned fiber) }\end{array}$ \\
\hline
\end{tabular}

Table 4

Convergence of elastic modulus using conforming tetra mesh type $\left(v_{g}=5 \%\right)$.

\begin{tabular}{llll}
\hline Element size & Number of elements & Number of nodes & $E_{11}(\mathrm{GPa})$ \\
\hline 0.080 & 33430 & 53404 & 3.329 \\
0.070 & 41943 & 66654 & 3.316 \\
0.060 & 59412 & 93467 & 3.309 \\
0.050 & 91402 & 142187 & 3.303 \\
0.040 & 151530 & 233324 & 3.303 \\
0.030 & 282486 & 430381 & 3.303 \\
\hline
\end{tabular}

different dispersion of graphene sheet including aligned and random orientations. It is due to fact that FE models accurately capture the Poisson's effect and all coupling effect occurred in micromechanical analysis of composite, such as extension-extension, shear-extension, shear-shear coupling. From Tables 7 and 8, it can be obvious that we can use these presented FE models for validating the analytical and experimental investigations.

In spite of fact that graphene nanofiller or its derivatives are difficult to incorporate uniformly or aligned in epoxy matrix, therefore, the random distribution with different shapes of fiber reinforcement also considered in this paper (shown in Fig. 7). The predicted results for elastic and shear moduli of cylindrical (C) and ellipsoidal (E) shaped graphene nanofillers reinforced to epoxy matrix using FE model for 1 $5 \%$ volume fraction of graphene is enlisted in Table 9. Due to cylindrical and ellipsoidal shape of inclusions, there are variations in effective axial, transverse and shear effective elastic properties of composite. There is a significant difference in geometrical shapes of cylindrical and ellipsoidal inclusions with respect to its volume fraction. In both cases, the effective properties increase with respect to the volume fraction of inclusions. By comparing Tables 6 and 9, it can be observed that the continuous fiber gives better results as compared to the inclusion type of fibers. In case of inclusion fibers, axial and transverse elastic moduli as well as in-plane and out-of-plane shear moduli are near about the same. In addition to this, the results for transverse elastic and shear modulus 
Table 5

Effective properties of GRC using MOM and FE model $\left(v_{g}=0.05-0.9 \%\right)$.

\begin{tabular}{|c|c|c|c|c|c|c|c|c|c|c|}
\hline \multirow[t]{2}{*}{ Volume fraction } & \multirow{2}{*}{$\frac{\mathrm{MOM}}{E_{11}(\mathrm{GPa})}$} & \multirow{2}{*}{$\frac{\mathrm{FE}}{E_{11}(\mathrm{GPa})}$} & \multirow{2}{*}{$\frac{\mathrm{MOM}}{E_{22}=E_{33}(\mathrm{GPa})}$} & \multirow{2}{*}{$\frac{\mathrm{FE}}{E_{22}=E_{33}(\mathrm{GPa})}$} & \multirow{2}{*}{$\frac{\mathrm{FE}}{\mu_{12}}$} & \multirow{2}{*}{$\frac{\mathrm{FE}}{\mu_{23}}$} & \multirow{2}{*}{$\frac{\mathrm{MOM}}{G_{12}(\mathrm{GPa})}$} & \multirow{2}{*}{$\frac{\mathrm{FE}}{G_{12}(\mathrm{GPa})}$} & \multirow{2}{*}{$\frac{\mathrm{FE}}{G_{23}(\mathrm{GPa})}$} & \multirow{2}{*}{$\frac{\mathrm{FE}}{\text { Density } \rho_{c}\left(\mathrm{~kg} / \mathrm{m}^{3}\right)}$} \\
\hline & & & & & & & & & & \\
\hline 0.05 & 3.302 & 3.303 & 2.890 & 2.888 & 0.2999 & 0.3161 & 1.097 & 1.097 & 1.097 & 1180 \\
\hline 0.1 & 3.800 & 3.802 & 2.921 & 2.920 & 0.2998 & 0.3298 & 1.097 & 1.098 & 1.098 & 1180 \\
\hline 0.112 & 3.865 & 3.866 & 2.928 & 2.924 & 0.2998 & 0.3312 & 1.097 & 1.099 & 1.098 & 1180 \\
\hline 0.2 & 4.742 & 4.744 & 2.966 & 2.965 & 0.2997 & 0.3482 & 1.098 & 1.101 & 1.100 & 1180 \\
\hline 0.3 & 5.689 & 5.691 & 2.997 & 2.998 & 0.2995 & 0.3607 & 1.099 & 1.103 & 1.102 & 1180 \\
\hline 0.4 & 6.610 & 6.611 & 3.021 & 3.022 & 0.2994 & 0.3696 & 1.101 & 1.105 & 1.103 & 1180 \\
\hline 0.5 & 7.548 & 7.551 & 3.039 & 3.042 & 0.2992 & 0.3765 & 1.102 & 1.107 & 1.105 & 1179 \\
\hline 0.6 & 8.490 & 8.492 & 3.054 & 3.059 & 0.2991 & 0.3819 & 1.103 & 1.109 & 1.107 & 1179 \\
\hline 0.7 & 9.429 & 9.432 & 3.066 & 3.073 & 0.2989 & 0.3863 & 1.104 & 1.111 & 1.108 & 1179 \\
\hline 0.8 & 1.032 & 1.037 & 3.077 & 3.086 & 0.2988 & 0.3898 & 1.105 & 1.114 & 1.110 & 1179 \\
\hline 0.9 & 1.128 & 1.131 & 3.087 & 3.097 & 0.2986 & 0.3928 & 1.106 & 1.116 & 1.112 & 1179 \\
\hline
\end{tabular}

Table 6

Effective properties of GRC using MOM and FE model $\left(v_{g}=1-10 \%\right)$.

\begin{tabular}{|c|c|c|c|c|c|c|c|c|c|c|}
\hline \multirow[t]{2}{*}{ Volume fraction } & \multirow{2}{*}{$\frac{\mathrm{MOM}}{E_{11}(\mathrm{GPa})}$} & \multirow{2}{*}{$\frac{\mathrm{FE}}{E_{11}(\mathrm{GPa})}$} & \multirow{2}{*}{$\frac{\mathrm{MOM}}{E_{22}=E_{33}(\mathrm{GPa})}$} & \multirow{2}{*}{$\frac{\mathrm{FE}}{E_{22}=E_{33}(\mathrm{GPa})}$} & \multirow{2}{*}{$\frac{\mathrm{FE}}{\mu_{12}}$} & \multirow{2}{*}{$\frac{\mathrm{FE}}{\mu_{23}}$} & \multirow{2}{*}{$\frac{\mathrm{MOM}}{G_{12}(\mathrm{GPa})}$} & \multirow{2}{*}{$\frac{\mathrm{FE}}{G_{12}(\mathrm{GPa})}$} & \multirow{2}{*}{$\frac{\mathrm{FE}}{G_{23}(\mathrm{GPa})}$} & \multirow{2}{*}{$\frac{\mathrm{FE}}{\text { Density } \rho_{c}\left(\mathrm{~kg} / \mathrm{m}^{3}\right)}$} \\
\hline & & & & & & & & & & \\
\hline 1 & 12.21 & 12.25 & 3.096 & 3.108 & 0.2985 & 0.3953 & 1.107 & 1.118 & 1.114 & 1179 \\
\hline 2 & 21.62 & 21.66 & 3.157 & 3.186 & 0.2970 & 0.4081 & 1.118 & 1.140 & 1.131 & 1178 \\
\hline 3 & 30.83 & 30.88 & 3.201 & 3.245 & 0.2955 & 0.4128 & 1.145 & 1.161 & 1.148 & 1176 \\
\hline 4 & 41.29 & 41.32 & 3.241 & 3.304 & 0.2939 & 0.4155 & 1.166 & 1.185 & 1.167 & 1175 \\
\hline 5 & 51.54 & 51.57 & 3.331 & 3.360 & 0.2924 & 0.4168 & 1.190 & 1.210 & 1.186 & 1174 \\
\hline 6 & 62.09 & 62.10 & 3.380 & 3.416 & 0.2908 & 0.4176 & 1.215 & 1.234 & 1.205 & 1173 \\
\hline 7 & 72.51 & 72.55 & 3.125 & 3.471 & 0.2892 & 0.4181 & 1.238 & 1.259 & 1.224 & 1172 \\
\hline 8 & 82.71 & 82.75 & 3.475 & 3.528 & 0.2877 & 0.4183 & 1.265 & 1.285 & 1.244 & 1170 \\
\hline 9 & 92.70 & 92.74 & 3.430 & 3.585 & 0.2862 & 0.4182 & 1.281 & 1.311 & 1.264 & 1169 \\
\hline 10 & 102.50 & 102.9 & 3.469 & 3.644 & 0.2847 & 0.4181 & 1.309 & 1.338 & 1.285 & 1168 \\
\hline
\end{tabular}

Table 7

Comparison of different models for effective elastic modulus of GRC ( $\left.v_{g}=0.1-0.5 \%\right)$.

\begin{tabular}{|c|c|c|c|c|c|c|c|c|}
\hline \multirow[t]{2}{*}{ Volume fraction (\%) } & \multirow[t]{2}{*}{ MOM } & \multicolumn{7}{|c|}{ Elastic modulus (GPa) } \\
\hline & & $\mathrm{FE}$ & FE (Inclusion) & ROM & $\begin{array}{l}\mathrm{mROM} \\
\eta_{0}=0.533\end{array}$ & $\mathrm{mROM} \eta_{0}=0.75$ & HT & Experimental \\
\hline 0.1 & 3.800 & 3.802 & 2.854 & 3.857 & 3.385 & 3.605 & 3.189 & \\
\hline 0.112 & 3.865 & 3.866 & 2.855 & 3.978 & 3.450 & 3.695 & 3.230 (Rafiee et al., 2009) & 3.740 (Rafiee et al., 2009) \\
\hline 0.2 & 4.742 & 4.744 & 2.862 & 4.864 & 3.921 & 4.359 & 3.528 & \\
\hline 0.3 & 5.689 & 5.691 & 2.869 & 5.871 & 4.456 & 5.114 & 3.867 & \\
\hline 0.4 & 6.610 & 6.611 & 2.875 & 6.879 & 4.992 & 5.869 & 4.206 & \\
\hline 0.5 & 7.548 & 7.551 & 2.873 & 7.886 & 5.527 & 6.623 & 4.545 & \\
\hline
\end{tabular}

for continuous fiber shows better coherence with results for transverse elastic and shear modulus in case of inclusion in composite. Unless otherwise mentioned, the results presented in Tables 7 and 9 for the composite with the incorporation of graphene inclusion nanofillers are in better agreement with the experimental outcomes presented in Table 8.

\subsection{Results of two- and three-phase MOM model}

In this section, we have considered a two-phase and hybrid threephase piezoelectric composite made of active piezoelectric PZT fibers, an epoxy matrix as well as with and without consideration of graphene nanofillers while PZT fibers aligned and poled parallel to each other along 1-direction of the laminate. In addition to this, we considered two types of two-phase composites such as graphene/epoxy composite (GRC) and PZT/epoxy composites (PRC). Thus, the results for two-phase GRC are plotted separately. The effective properties of two-phase GRC are computed by varying volume fractions of graphene nanofillers $\left(v_{g}\right)$. Fig. 8 shows the variation of different effective elastic stiffness coefficients with respect to $v_{g}$. It shows a significant enhancement in the properties of pure epoxy due to addition of graphene in the percentage of $0.1-1 \%$ volume fraction in composite.

The effective properties of three-phase graphene reinforced piezoelectric composite (GRPC) are computed by varying volume fractions of graphene interphase $\left(v_{g}\right)$ and PZT fibers $\left(v_{p}\right)$. In this work, we considered distinct percentages of graphene nanofillers with respect to $v_{p}$ such as: $0.75 v_{p}, 0.50 v_{p}$ and $0.25 v_{p}$ for three-phase GRPC while in twophase PRC, an addition of graphene nanofillers are not considered. All sets of effective piezoelastic $\left(C_{11}, C_{12}, C_{13}, C_{23}, C_{33}, C_{66}, e_{31}, e_{32}, e_{33}\right)$ properties of GRPC are determined by considering the iso-stress/strain conditions and ROM. Using Eq. (14), values of all effective piezoelastic constants are estimated for any combination of $v_{p}$ and $v_{g}$. Fig. 9 depicts the variation of $C_{11}$ with respect to the volume fraction of PZT fiber and graphene interphase for two- and three-phase composite. The values of $C_{11}$ show linear variation with respect to $v_{p}$ and $v_{g}$ which is due to fact that it perfectly follows iso-stress/strain conditions (Eq. (10)) and exactly fits with the Voigt-upper bound and experimental findings (as shown in Table 6 for $0.112 \%$ ). It is noticed from Fig. 8 that due to the incorporation of graphene nanofillers, the value of $C_{11}$ for the GRPC improves as that of two-phase PRC. Figs. 10-13 illustrate the comparison between the set of results for transverse elastic constants $\left(C_{12}, C_{13}\right.$, $C_{23}, C_{33}$ ) for two- and three-phase composite. These results do not show 
Table 8

Comparison of existing experimental prediction of effective elastic modulus of GRC with FE models considering aligned and randomly distributed graphene sheet (GS) for different volume or weight fraction.

\begin{tabular}{|c|c|c|c|c|}
\hline \multicolumn{5}{|c|}{ Elastic modulus (GPa) [vol\% (wt.\%)] } \\
\hline $\begin{array}{l}\text { Present results } \\
\text { (vol\%) }\end{array}$ & $\begin{array}{l}\text { (Rafiee et al., } \\
\text { 2010) wt. (\%) }\end{array}$ & $\begin{array}{l}\text { (Zaman et al., } \\
\text { 2011, 2012) } \\
\text { vol. (\%) }\end{array}$ & $\begin{array}{l}\text { (Tang et al., } \\
\text { 2013) [vol\% } \\
\text { (wt.\%)] }\end{array}$ & $\begin{array}{l}\text { Rafiee } \\
\text { et al. } \\
(2009)\end{array}$ \\
\hline $\begin{array}{l}3.866(\mathrm{FE}- \\
0.112) \\
3.865 \\
(\mathrm{MOM}- \\
0.112) \\
\text { Aligned GS }\end{array}$ & - & $2.95(0.244 \%)$ & $\begin{array}{l}\sim 3.10 \\
{[0.025} \\
(0.05)]\end{array}$ & $\begin{array}{l}\text { Aligned } \\
\text { GS } \\
3.740 \\
{[0.112} \\
(0.10)]\end{array}$ \\
\hline $\begin{array}{l}2.865(\mathrm{FE}- \\
0.25) \\
2.873(\mathrm{FE}-\end{array}$ & $\sim 3.00(0.05)$ & $2.92(0.489 \%)$ & $\begin{array}{l}\sim 3.15 \\
{[0.051} \\
(0.10)]\end{array}$ & \\
\hline $\begin{array}{l}0.5) \\
2.978(\mathrm{FE} \\
-1)\end{array}$ & $\sim 4.1(0.10)$ & $3.01(0.984 \%)$ & $\begin{array}{l}\sim 3.20 \\
{[0.102} \\
(0.20)]\end{array}$ & \\
\hline $\begin{array}{l}3.024 \text { (FE } \\
-2 \text { ) } \\
\text { Randomly } \\
\text { distributed } \\
\text { GS }\end{array}$ & $\begin{array}{l}\sim 4.00(0.125) \\
\text { Functionalized } \\
\text { Aligned GS }\end{array}$ & $\begin{array}{l}3.26(1.988 \%) \\
\text { Randomly } \\
\text { distributed GS }\end{array}$ & $\begin{array}{l}- \\
\text { Highly } \\
\text { dispersed } \\
\text { graphene }\end{array}$ & \\
\hline
\end{tabular}

the linear variation which is due to fact that the transverse elastic constants of two- and three-phase composite are mainly matrix dominant. These constants signify the extension-extension coupling (Poisson's effect) which exists amongst the distinct normal stresses and strains due to application of load in the fiber direction. Due to consideration of axis of symmetry along the fiber direction, the values of $C_{22}$ and $C_{33}$ are approximately same. The effective properties of composite are predicted with and without considering strong covalent bond which provide interaction and in-plane stability of 2Dcrystalline graphene or strong van der Wall forces formed between graphene layers and the matrix. It is observed that the axial, transverse and shear effective piezoelastic properties of GRPC are significantly enhanced due to the incorporation of graphene into the epoxy matrix. Fig. 14 shows the variation of values of in-plane and out-of-plane shear constants $\left(C_{44}, C_{55}\right.$ and $\left.C_{66}\right)$ with respect to $v_{p}$ and $v_{g}$ for two- and three-phase composite. The micromechanical model does not account boundary conditions and thus gives similar results for in-plane and out-of-plane shear constants and hence, results for $C_{66}$ is only presented here. In addition to this, these effective shear elastic constants are dependent on axial and transverse elastic constants. The estimations of $C_{44}, C_{55}$ and $C_{66}$ are purely based on shear boundary conditions applied to the RVE of GRPC which can be considered in FE model. For the sake of brevity, the results for FE model are not presented in these plots of Figs. 9-14, but both models are found to be in good agreement. For the better understanding of readers, FE procedure for determination of the effective elastic constant $C_{22}$ of PRC or GRPC is already explained in section 3.

From Figs. 9-14, it is observed that all the effective elastic stiffness constants of three-phase composite are much greater as compared to the previously reported two-phase PRC (Kumar and Chakraborty, 2009; wherein they have taken large value of epoxy i.e., approximately $\sim 4 \mathrm{GPa}$ as we estimated results for the value of epoxy is $2.85 \mathrm{GPa}$ ). From the estimated values of above elastic stiffness constants, it is clearly seen that the results for the three-phase GRPC are significantly enhanced due to incorporation of graphene interphase which is nearly two/three times as compared to the two-phase PRC i.e., without consideration of graphene interphase for $v_{p}=0.4$. The main reason behind these improved properties is due to the fact that the graphene is having large surface area for interaction with fiber and matrix.

Figs. 15-17 depict the results for effective piezoelectric constants $\left(e_{31}, e_{32}\right.$ and $\left.e_{33}\right)$ of two- and three-phase composite with respect to volume fraction of PZT and graphene. Due to application of electric field across the thickness of composite, the effective piezoelectric constants $e_{31}$ and $e_{32}$ proportional to the in-plane actuation while $e_{33}$ proportional to the out-of-plane actuations, respectively. The effective piezoelastic properties of GRPC are determined by considering the uniform stress and strain conditions. It is observed from Fig. 15 that the values of $e_{31}$ increase with the values $v_{p}$ and $v_{g}$. It can be noted that the values of $e_{31}$ shows the linear variation with respect to values of $v_{p}$ and are related to

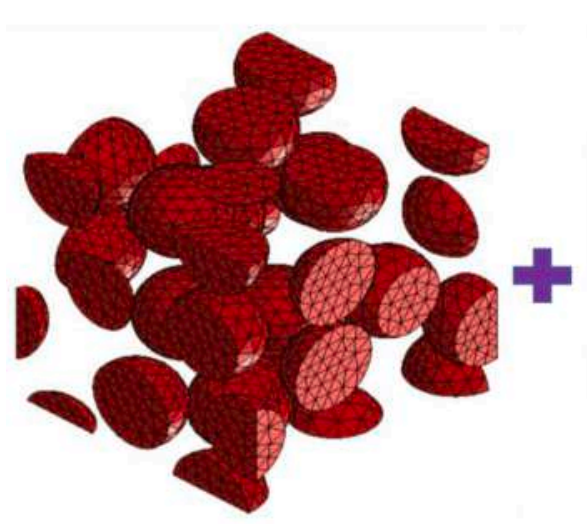

Fiber

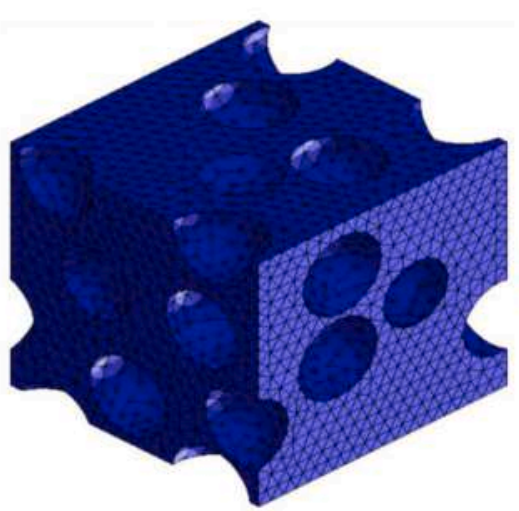

Matrix

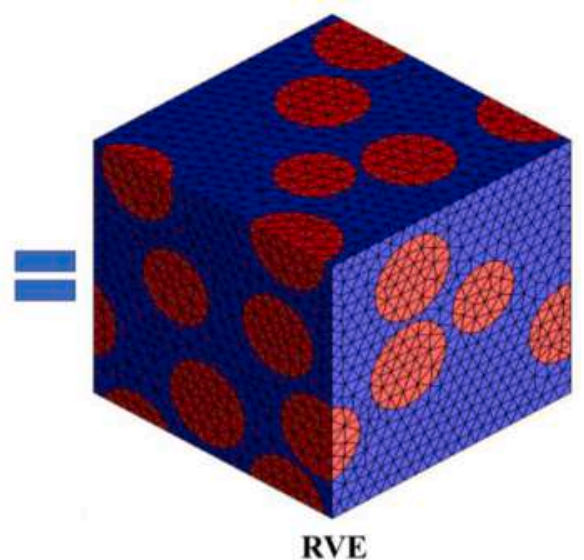

RVE

Fig. 7. 3Dschematic representation of randomly distributed ellipsoidal shaped fiber RVE.

Table 9

Elastic and shear modulus of cylindrical (C) and ellipsoidal (E) shaped graphene nanofillers reinforced to epoxy matrix using FE model $\left(v_{g}=1-5 \%\right)$.

\begin{tabular}{|c|c|c|c|c|c|c|c|c|c|c|c|c|}
\hline \multirow[t]{2}{*}{ Volume fraction (\%) } & \multicolumn{2}{|c|}{$E_{11}(\mathrm{GPa})$} & \multicolumn{2}{|c|}{$E_{22}(\mathrm{GPa})$} & \multicolumn{2}{|c|}{$E_{33}(\mathrm{GPa})$} & \multicolumn{2}{|c|}{$G_{12}(\mathrm{GPa})$} & \multicolumn{2}{|c|}{$G_{23}(\mathrm{GPa})$} & \multicolumn{2}{|c|}{$G_{13}(\mathrm{GPa})$} \\
\hline & $\mathrm{C}$ & $\mathrm{E}$ & $\mathrm{C}$ & $\mathrm{E}$ & $\mathrm{C}$ & $\mathrm{E}$ & $\mathrm{C}$ & $\mathrm{E}$ & $\mathrm{C}$ & $\mathrm{E}$ & $\mathrm{C}$ & $\mathrm{E}$ \\
\hline 1 & 2.978 & 2.961 & 2.920 & 2.961 & 2.920 & 2.905 & 1.122 & 1.119 & 1.123 & 1.118 & 1.122 & 1.119 \\
\hline 2 & 3.024 & 2.965 & 2.994 & 2.964 & 2.992 & 2.967 & 1.148 & 1.143 & 1.152 & 1.142 & 1.149 & 1.142 \\
\hline 3 & 3.122 & 3.021 & 3.068 & 3.022 & 3.075 & 3.022 & 1.178 & 1.165 & 1.180 & 1.169 & 1.177 & 1.167 \\
\hline 4 & 3.224 & 3.089 & 3.141 & 3.084 & 3.147 & 3.090 & 1.204 & 1.190 & 1.207 & 1.190 & 1.210 & 1.217 \\
\hline 5 & 3.302 & 3.153 & 3.224 & 3.153 & 3.220 & 3.147 & 1.232 & 1.218 & 1.236 & 1.219 & 1.229 & 1.219 \\
\hline
\end{tabular}



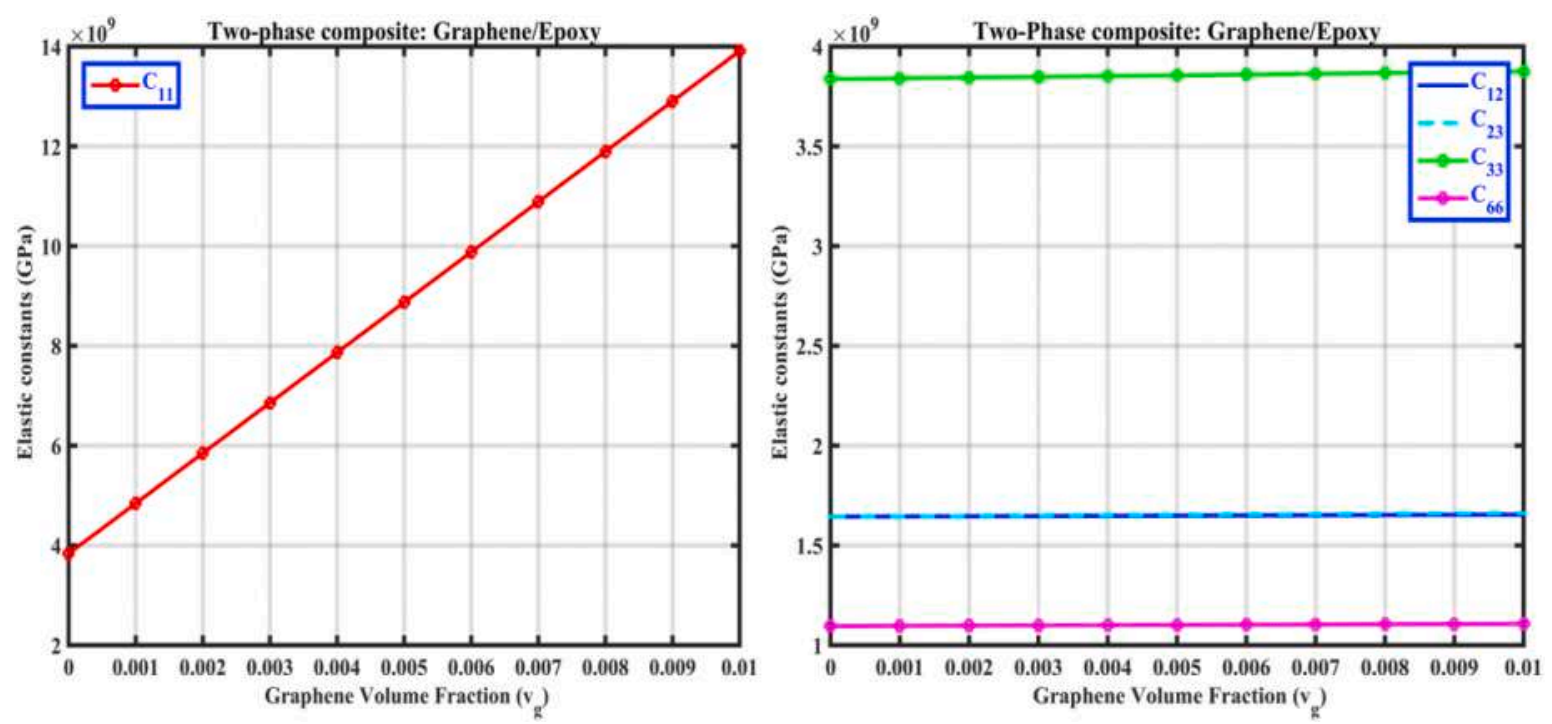

Fig. 8. Variation of elastic stiffness coefficients with respect to $v_{g}$ for two-phase composite.

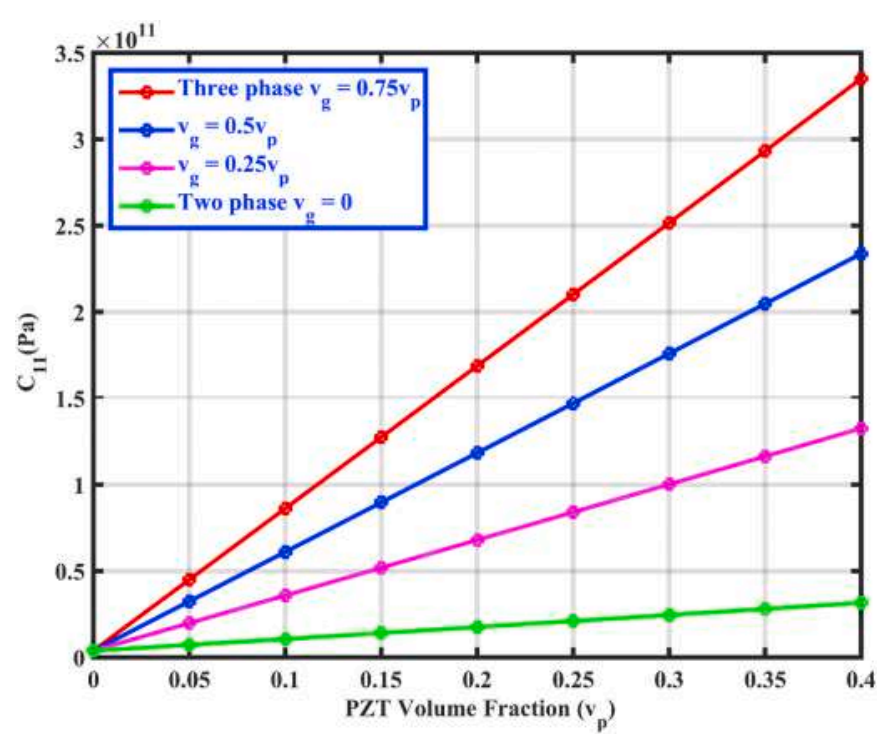

Fig. 9. Variation of $C_{11}$ with respect to $v_{p}$ and $v_{g}$ for two- and threephase composite.

the Voigt-upper bound estimation. It is also clearly seen from Fig. 15 that the values of $e_{31}^{p}$ for PZT are greater than the estimated values of $e_{31}$ for GRPC at higher value of $v_{p}$, but for the sake brevity, values up to $v_{p}=0.4$ are presented here. It is due to the influence of transverse stresses applied by the epoxy matrix on the GRPC and the definite average electric field in PZT becomes same as that of applied electric field. From Fig. 16, it is also found that the value of another in-plane effective piezoelectric constant $e_{32}$ also increases with respect to $v_{p}$ and $v_{g}$. From Fig. 17, it is clearly seen that the results for piezoelectric constants $e_{33}$ of the GRPC are significantly enhanced due to incorporation of graphene interphase which is nearly two times as compared to the two-phase PRC i.e., without consideration of interphase for $v_{p}=0.4$. Also, it can be observed from Figs. 15-17 that the values of the effective piezoelectric constants increase with an increase in value of $v_{g}$. Though, incorporation of graphene nanofillers does not influence the value of $e_{31}$. It is found that the values of $e_{33}$ for GRPC are significantly greater as compared to the previously reported PRC (Kumar and Chakraborty, 2009) at the practically suitable value of $v_{p}$. Because of increase in the value of $e_{33}$,

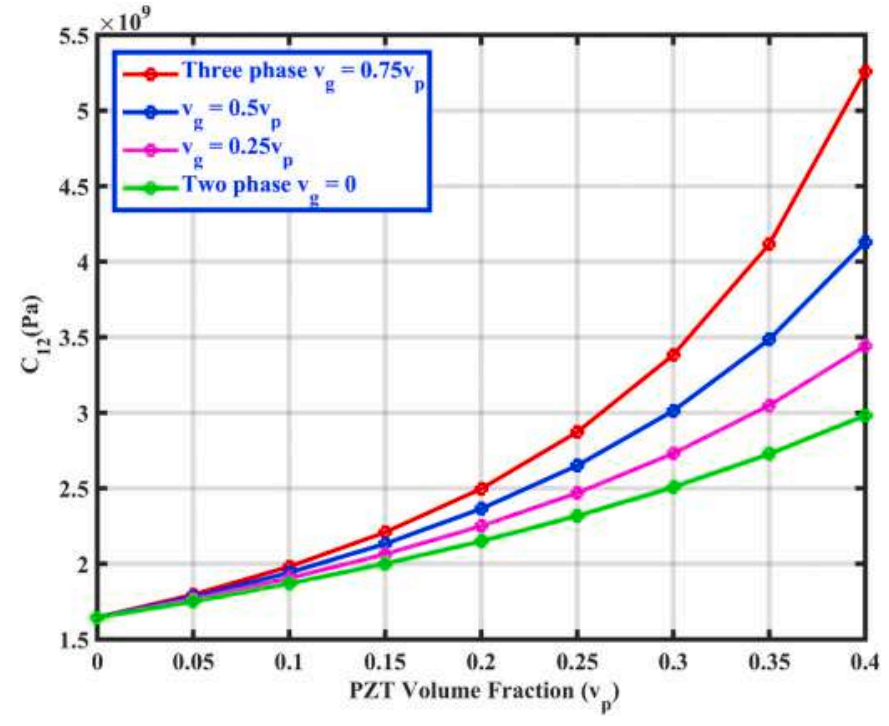

Fig. 10. Variation of $C_{12}$ with respect to $v_{p}$ and $v_{g}$ for two- and threephase composite.

for both in-plane and out-of-plane actuations, these three-phase composite can be served as a distributed actuator while the in-plane actuation reported by earlier study for two-phase composites is marginally insignificant.

The substantial enhancement observed in the effective properties of the GRPC is due to fact that the graphene interphase reinforcements improve the properties of the matrix. The comparative results presented in Figs. 9-17 show that due to incorporation of graphene nanofillers, the noteworthy enrichment can be observed in all piezoelastic constants. Hence it can be concluded that the work presented in this paper can be served as a pathway for emerging new effective smart structures with an enriched control authority.

\section{Concluding remarks}

In this article, the different micromechanical modelling techniques based on analytical and numerical approaches are studied to determine the effective elastic and piezoelectric properties of graphene-based composites such as GRCs and GRPCs. The outcomes presented in this 


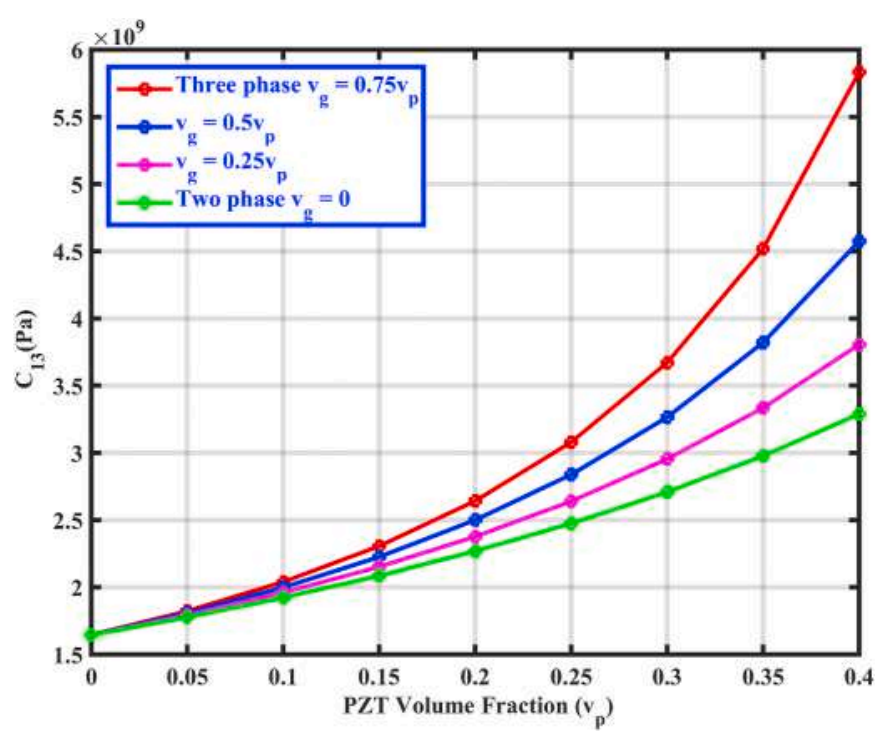

Fig. 11. Variation of $C_{13}$ with respect $v_{p}$ and $v_{g}$ for two- and threephase composite.

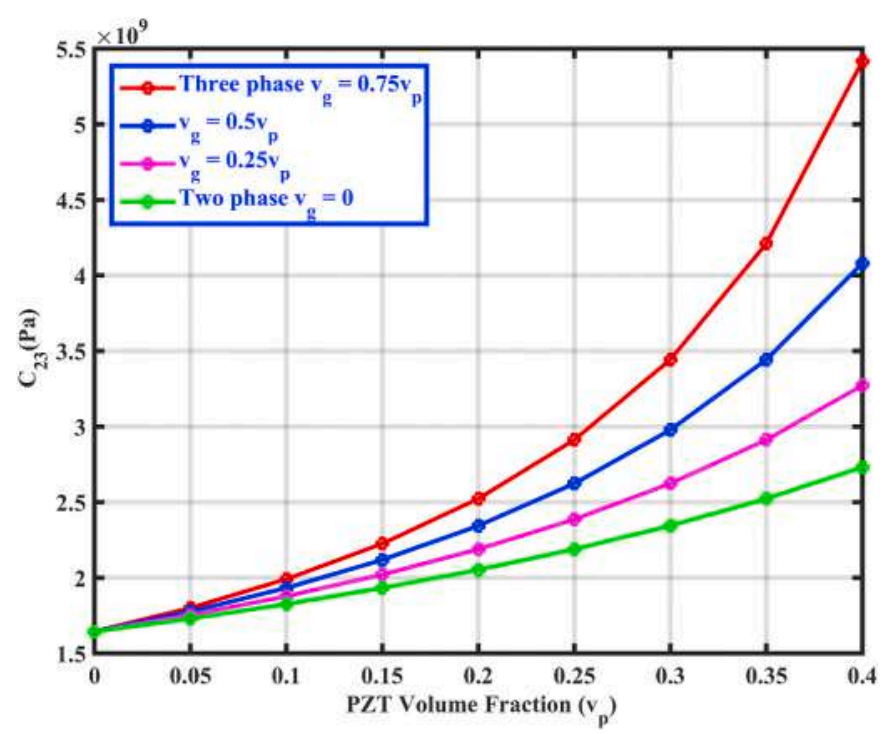

Fig. 12. Variation of $C_{23}$ with respect $v_{p}$ and $v_{g}$ for two- and threephase composite.

paper offer a thorough insight on the effects of incorporation of graphene nanofillers in the effective properties of composite considering different techniques such as: ROM, mROM, HT method, MOM and FE methods with different shape of fibers including continuous fiber, cylindrical and ellipsoidal inclusions. In case of $\mathrm{mROM}$, different factors including Krenchel orientation $\left(\eta_{0}\right)$, critical length efficiency $\left(\eta_{l}\right)$ and agglomeration $\left(\eta_{a}\right)$ factors play significant role while evaluating the effective properties of graphene-based composites while the geometrical parameters are considered in HT model. The effective properties of composite are predicted with and without considering strong covalent bond which provides interaction and in-plane stability of $2 D$ crystalline graphene or strong van der Wall forces formed between graphene layers and the matrix. From above studies, it is concluded that the random orientation of fiber reinforcement gives noteworthy results as incorporation of nanofillers aligned or uniformly in matrix is difficult to achieve and resembles with experimental estimates. It is observed that the axial, transverse and shear effective piezoelastic properties of GRPC are significantly enriched due to the incorporation of graphene into the

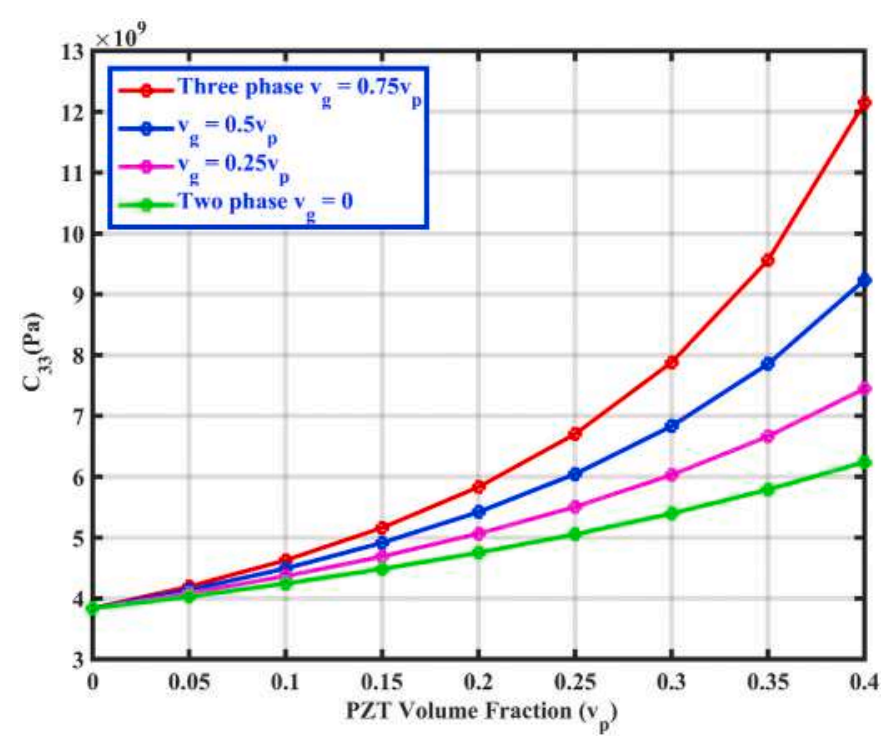

Fig. 13. Variation of $C_{33}$ with respect to $v_{p}$ and $v_{g}$ for two- and threephase composite.

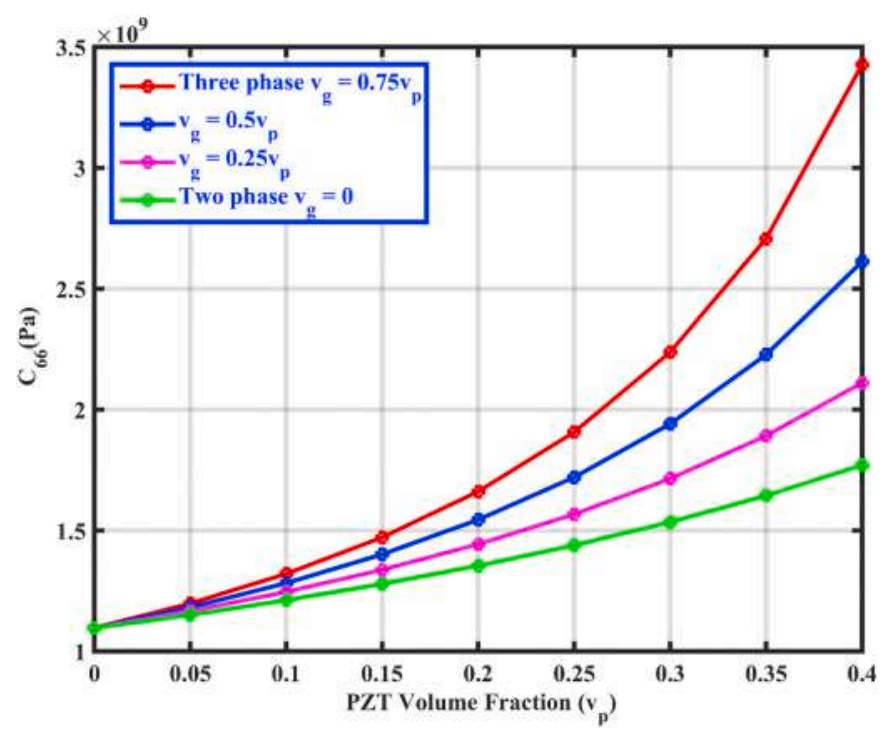

Fig. 14. Variation of $C_{66}$ with respect to $v_{p}$ and $v_{g}$ for two- and threephase composite.

epoxy matrix. It is also revealed that the large surface area of graphene helps in strong interaction with fiber and matrix which results in improved effective properties. In addition to this, the significant results presented in the paper, for elastic and shear effective properties of the composite are observed in case of cylindrical and ellipsoidal shaped nanofillers. The results predicted using different micromechanical models such as ROM, mROM, HT and present FE numerical models are validated using experimental results reported in the existing literature. Outcome presented in this study will have a significant influence on the current design procedures in the dynamic and structural analyses of various advanced composite structures.

Therefore, it is imperative to account the effect of influence of graphene as fiber or nanofillers to ensure the desired sustainable and robust system performance. The novelty of the work presented here, comprises the consideration of graphene nanofillers in effective properties of composite following the different FE models via RVE based approach. It can be obvious that one can use these FE models for validating and bridging the gap between the theoretical and experimental studies and 


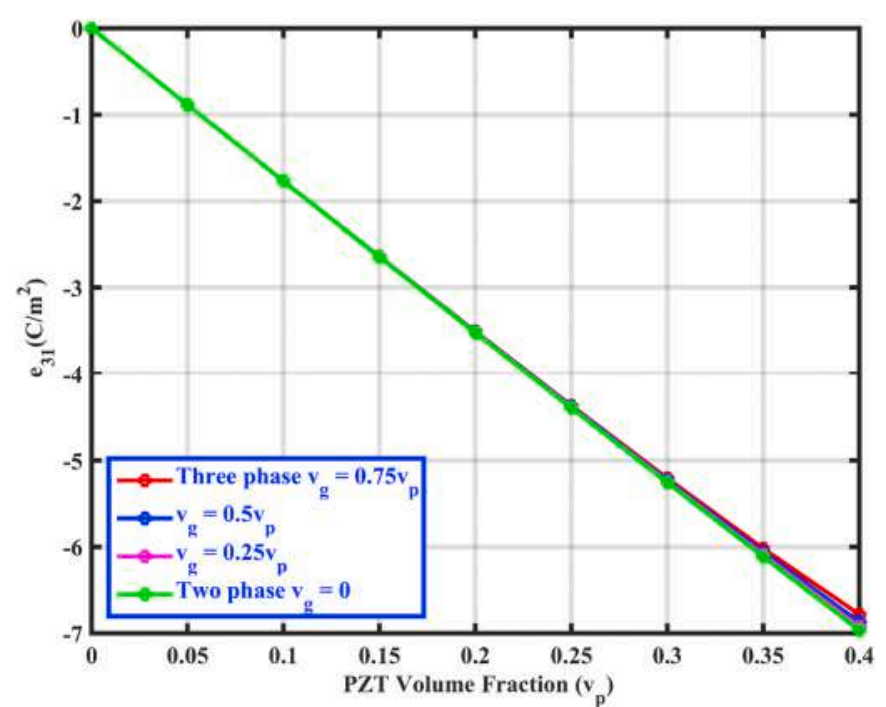

Fig. 15. Variation of $e_{31}$ with respect to $v_{p}$ and $v_{g}$ for two and threephase composite.

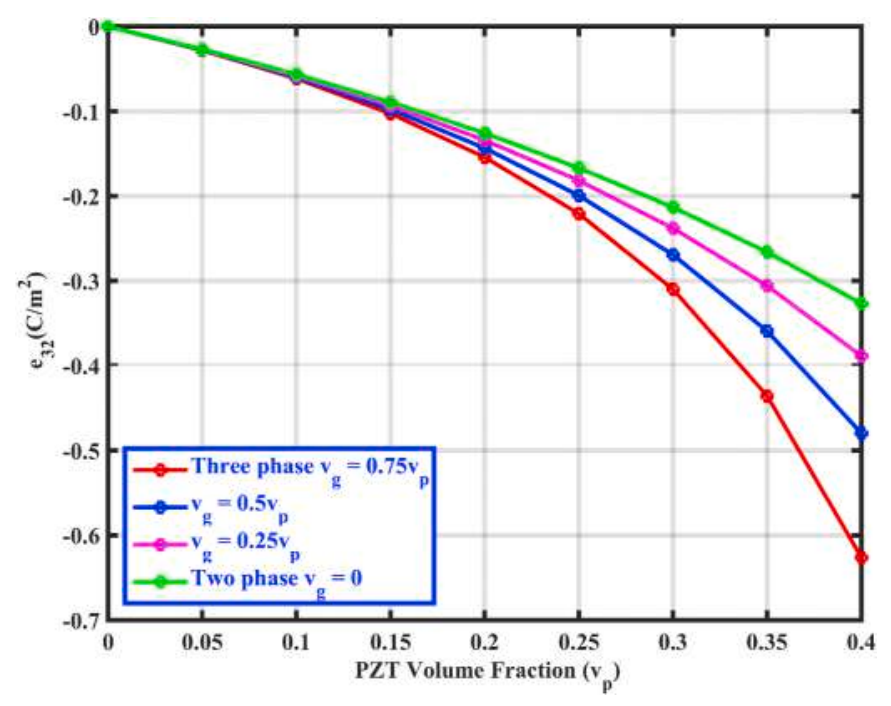

Fig. 16. Variation of $e_{32}$ with respect to $v_{p}$ and $v_{g}$ for two and threephase composite.

can also be utilized in real life industrial applications. The interphase effects can help to overcome the basic nanofiller influence and tend to greater stiffnesses for any configurations of graphene-reinforced composites. Even if several complexities are ignored, these presented comparative results can offer comprehensive guidance to evaluate experimental data and to design nanocomposite made of graphene and graphene-like2Dnanomaterials as reinforcing phase or as interphase. These findings inspire and provide a basis for future experimentation with different sized and shaped nanofillers in the epoxy matrix, which are presently absent in the literature. These simple but insightful comparative studies can offer pathways to the advanced and multifunctional design of nanocomposites. The concept of RVE with continuous fiber is quite general in nature, hence it can be extended to other structures with two and three dimensions. Moreover, the proposed effective properties can be utilized in various thin-walled composite structures such as beam, plate and shell for illustrating various global responses in future.

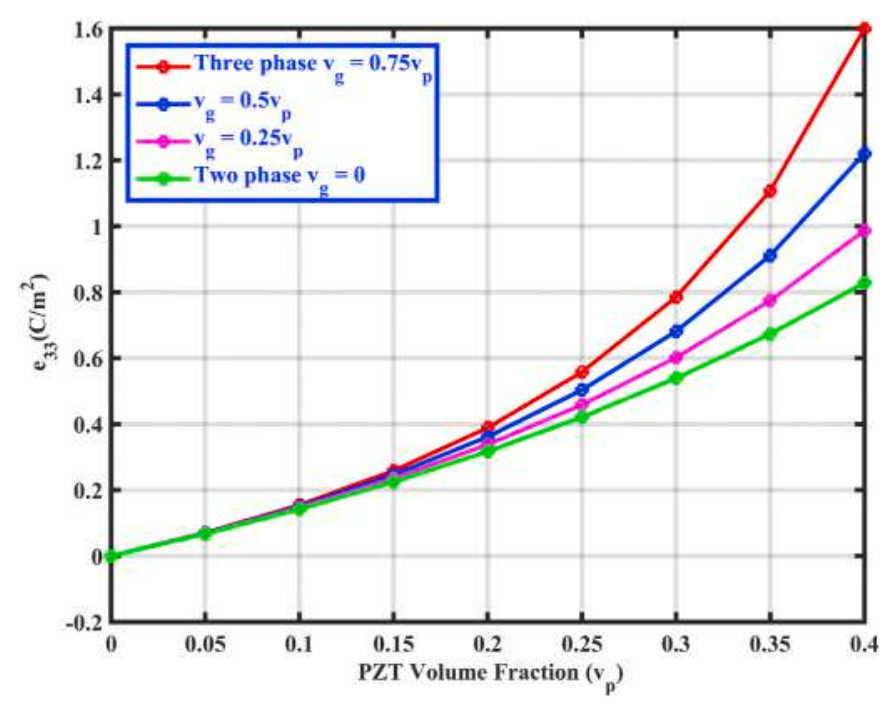

Fig. 17. Variation of $e_{33}$ with respect to $v_{p}$ and $v_{g}$ for two and threephase composite.

\section{Declaration of competing interest}

The authors declare that they have no known competing financial interests or personal relationships that could have appeared to influence the work reported in this paper.

\section{Acknowledgement}

KBS acknowledges institute post-doctoral fellowship received from IIT Bombay. SN acknowledges the initiation grants received from IIT Bombay.

\section{References}

Affdl, J.C.H., Kardos, J.L., 1976. The Halpin-Tsai equations: a review. Polym. Eng. Sci. 16 (5), 344-352. https://doi.org/10.1002/pen.760160512.

Ansari, M.I., Chaubey, A.K., Kumar, A., Chakrabarti, A., Mishra, S.S., 2019. Analysis of functionally graded carbon nanotube-reinforced laminates. Mater. Today: Proceedings 18, 628-637. https://doi.org/10.1016/j.matpr.2019.06.457.

Benveniste, Y., Dvorak, G.J., 1992. Uniform fields and universal relations in piezoelectric composites. J. Mech. Phys. Solid. 40, 1295-1312. https://doi.org/10.1016/00225096(92)90016-U.

Berger, H., Kari, S., Gabbert, U., Rodriguez-Ramos, R., Bravo-Castillero, J., GuinovartDiaz, R., 2005. Calculation of effective coefficients for piezoelectric fiber composites based on a general numerical homogenization technique. Compos. Struct. 71, 397-400. https://doi.org/10.1016/j.compstruct.2005.09.038.

Berger, H., Kari, S., Gabbert, U., Rodríguez Ramos, R., Bravo Castillero, J., Guinovart Díaz, R., 2007. Evaluation of effective material properties of randomly distributed short cylindrical fiber composites using a numerical homogenization technique. J. Mech. Mater. Struct. 2, 1561-1570. https://doi.org/10.2140/jomms.2007.2-8.

Chandra, Y., Chowdhury, R., Scarpa, F., Adhikari, S., Sienz, J., Arnold, C., Murmu, T., Bould, D., 2012. Vibration frequency of graphene based composites: a multiscale approach. Mater. Sci. Eng., B 177, 303-310. https://doi.org/10.1016/j. mseb.2011.12.024.

Chen, L., Jin, H., Xu, Z., Shan, M., Tian, X., Yang, C., Wang, Z., Cheng, B., 2014. A design of gradient interphase reinforced by silanized graphene oxide and its effect on carbon fiber/epoxy interface. Mater. Chem. Phys. 145, 186-196. https://doi.org/ 10.1016/j.matchemphys.2014.02.001.

Deierling, P.E., Zhupanska, O.I., 2018. Computational modeling of the effective properties of spatially graded composites. Int. J. Mech. Sci. 145, 145-157. https:// doi.org/10.1016/j.ijmecsci.2018.06.029.

Dey, S., Adhikari, S., Karmakar, A., 2015a. Impact response of functionally graded conical shells. Lat. Am. J. Solid. Struct. 12 (1), 133-152. https://doi.org/10.1590/ 1679-78251382.

Dey, S., Sarkar, S., Das, A., Karmakar, A., Adhikari, S., 2015b. Effect of twist and rotation on vibration of functionally graded conical shells. Int. J. Mech. Mater. Des. 11 (4), 425-437. https://doi.org/10.1007/s10999-014-9266-x.

Dey, S., Naskar, S., Mukhopadhyay, T., Gohs, U., Spickenheuer, A., Bittrich, L., Sriramula, S., Adhikari, S., Heinrich, G., 2016. Uncertain natural frequency analysis of composite plates including effect of noise - A polynomial neural network approach. Compos. Struct. 143, 130-142. https://doi.org/10.1016/j. compstruct.2016.02.007. 
Dey, S., Mukhopadhyay, T., Naskar, S., Dey, T.K., Chalak, H.D., Adhikari, S., 2019. Probabilistic characterisation for dynamics and stability of laminated soft core sandwich plates. J. Sandw. Struct. Mater. 21 (1) https://doi.org/10.1177/ 1099636217694229.

Du, X., Skachko, I., Barker, A., Andrei, E.Y., 2008. Approaching ballistic transport in suspended graphene. Nat. Nanotechnol. 3, 491-495. https://doi.org/10.1038/ nnano.2008.199.

E-xstream.com, 2018. Digimat, the Material Modeling Platform.

Esteva, M., Spanos, P., 2009. Effective elastic properties of nanotube reinforced composites with slightly weakened interfaces. J. Mech. Mater. Struct. 4 (5), 887-900. https://doi.org/10.2140/jomms.2009.4.887.

Fantuzzi, N., Bacciocchi, M., Agnelli, J., Benedetti, D., 2020. Three-phase homogenization procedure for woven fabric composites reinforced by carbon nanotubes in thermal environment. Compos. Struct. 254, 112840. https://doi.org/ 10.1016/j.compstruct.2020.112840.

Gopinath, G., Batra, R.C., 2018. A common framework for three micromechanics approaches to analyze elasto-plastic deformations of fiber-reinforced composites. Int. J. Mech. Sci. 148, 540-553. https://doi.org/10.1016/j.ijmecsci.2018.09.003.

Greco, F., Luciano, R., 2011. A theoretical and numerical stability analysis for composite micro-structures by using homogenization theory. Compos. B Eng. 42, 382-401. https://doi.org/10.1016/j.compositesb.2010.12.006.

Guzmán de Villoria, R., Miravete, A., 2007. Mechanical model to evaluate the effect of the dispersion in nanocomposites. Acta Mater. 55, 3025-3031. https://doi.org/ 10.1016/j.actamat.2007.01.007.

Hagood, N., Bent, A., 1993. Development of Piezoelectric Fiber Composites for Structural Actuation. 34th Structures, Structural Dynamics and Materials Conference, p. 1717. https://doi.org/10.2514/6.1993-1717.

Halpin, J., 1969. Effects of environmental factors on composite materials. Air Force Materials Lab Wright-Patterson AFB OH. https://doi.org/10.1016/j. compscitech.2008.01.014.

Halpin, J.C., Thomas, R.L., 1968. Ribbon reinforcement of composites. J. Compos. Mater. 2 (4), 488-497. https://doi.org/10.1177/002199836800200409.

Hashin, Z., 1990. Thermoelastic properties of fiber composites with imperfect interface. Mech. Mater. 8 (4), 333-348. https://doi.org/10.1016/0167-6636(90)90051-G.

Hashin, Z., Shtrikman, S., 1962a. On some variational principles in anisotropic and nonhomogeneous elasticity. J. Mech. Phys. Solid. 10, 335-342. https://doi.org/ 10.1016/0022-5096(62)90004-2.

Hashin, Z., Shtrikman, S., 1962b. A variational approach to the theory of the elastic behaviour of polycrystals. J. Mech. Phys. Solid. 10, 343-352. https://doi.org/ 10.1016/0022-5096(62)90005-4.

Hill, R., 1952. The elastic behaviour of a crystalline aggregate. Proc. Phys. Soc. 65, 349-354. https://doi.org/10.1088/0370-1298/65/5/307.

Karakassides, A., Ganguly, A., Tsirka, K., Paipetis, A.S., Papakonstantinou, P., 2020. Radially grown graphene nanoflakes on carbon fibers as reinforcing interface for polymer composites. ACS Appl. Nano Mater. 3 (3), 2402-2413. https://doi.org/ 10.1021/acsanm.9b02536.

Kari, S., Berger, H., Rodriguez-Ramos, R., Gabbert, U., 2007. Computational evaluation of effective material properties of composites reinforced by randomly distributed spherical particles. Compos. Struct. 77 (2), 223-231. https://doi.org/10.1016/j. compstruct.2005.07.003.

Karsh, P.K., Mukhopadhyay, T., Chakraborty, S., Naskar, S., Dey, S., 2019. A hybrid stochastic sensitivity analysis for low-frequency vibration and low-velocity impact of functionally graded plates. Compos. Part B: Eng. 176 https://doi.org/10.1016/j. compositesb.2019.107221.

Kashtalyan, M., 2004. Three-dimensional elasticity solution for bending of functionally graded rectangular plates. Eur. J. Mech. A Solids 23 (5), 853-864. https://doi.org/ 10.1016/j.euromechsol.2004.04.002.

Kumar, A., Chakraborty, D., 2009. Effective properties of thermo-electro-mechanically coupled piezoelectric fiber reinforced composites. Mater. Des. 30 (4), 1216-1222. https://doi.org/10.1016/j.matdes.2008.06.009.

Kumar, R.R., Mukhopadhyay, T., Naskar, S., Pandey, K.M, Dey, S., 2019. Stochastic lowvelocity impact analysis of sandwich plates including the effects of obliqueness and twist. Thin-Walled Struct. 145 https://doi.org/10.1016/j.tws.2019.106411.

Kundalwal, S.I., Shingare, K.B., Gupta, M., 2020a. Flexoelectric effect on electri potential in piezoelectric cylindrical graphene-based composite nanowire: Analytical and numerical modelling. Euro. J. Mech. A/Sol. 84, 104050.

Kundalwal, S.I., Shingare, K.B., Maware, P.P., 2020b. Carbon fiber reinforced nanocomposites: a multiscale modeling of regularly staggered carbon fibers, fiberreinforced nanocomposites: fundamentals and applications. Micro Nano Technol. 101-127. https://doi.org/10.1016/B978-0-12-819904-6.00005-0.

Kundalwal, S.I., Shingare, K.B., Rathi, A., 2019. Effect of flexoelectricity on the electromechanical response of graphene nanocomposite bea. Int. J. Mech. Mater. Des. 15, 447-470. https://doi.org/10.1007/s10999-018-9417-6.

Li, Z., Chu, J., Yang, C., Hao, S., Bissett, M.A., Kinloch, I.A., Young, R.J., 2018. Effect of functional groups on the agglomeration of graphene in nanocomposites. Compos. Sci. Technol. 163, 116-122. https://doi.org/10.1016/j.compscitech.2018.05.016.

Luciano, R., Tamburrino, A., 2000. Homogenization techniques for linear dielectric random composite materials in stationary conditions. Int. J. Appl. Electromagn. Mech. 11, 163-178. https://doi.org/10.3233/jae-2000-179.

Martínez-Ayuso, G., Friswell, M.I., Adhikari, S., Khodaparast, H.H., Berger, H., 2017. Homogenization of porous piezoelectric materials. Int. J. Solid Struct. 113 (114), 218-229. https://doi.org/10.1016/j.ijsolstr.2017.03.003.

Mirabedini, A., Ang, A., Nikzad, M., Fox, B., Lau, K.T., Hameed, N., 2020. Evolving strategies for producing multiscale graphene-enhanced fiber-reinforced polymer composites for smart structural applications. Adv. Sci. 7, 1903501. https://doi.org/ 10.1002/advs.201903501.
Naskar, S., Mukhopadhyay, T., Sriramula, S., 2019. Spatially varying fuzzy multi-scale uncertainty propagation in unidirectional fibre reinforced composites. Compos. Struct. 209, 940-967. https://doi.org/10.1016/j.compstruct.2018.09.090.

Mukhopadhyay, T., Naskar, S., Dey, S., Adhikari, S., 2016. On quantifying the effect of noise in surrogate based stochastic free vibration analysis of laminated composite shallow shells. Compos. Struct. 140, 798-805. https://doi.org/10.1016/j. compstruct.2015.12.037.

Mukhopadhyay, T., Naskar, S., Karsh, P.K., Dey, S., You, Z., 2018. Effect of delamination on the stochastic natural frequencies of composite laminates. Compos. Part B: Eng. 154, 242-256. https://doi.org/10.1016/j.compositesb.2018.07.029.

Mukhopadhyay, T., Naskar, S., Chakraborty, S., Karsh, P.K., Choudhury, R., Dey, S., 2020. Stochastic oblique impact on composite laminates: a concise review and characterization of the essence of hybrid machine learning algorithms. Arch. Comput. Methods Eng. https://doi.org/10.1007/s11831-020-09438-w.

Naskar, S., Mukhopadhyay, T., Sriramula, S., 2018. Probabilistic micromechanical spatial variability quantification in laminated composites. Compos. B Eng. 151, 291-325. https://doi.org/10.1016/j.compositesb.2018.06.002.

Naskar, S., Mukhopadhyay, T., Sriramula, S., Adhikari, S., 2017. Stochastic natural frequency analysis of damaged thin-walled laminated composite beams with uncertainty in micromechanical properties. Compos. Struct. 160, 312-334. https:// doi.org/10.1016/j.compstruct.2016.10.035.

Novoselov, K.S., Geim, A.K., Morozov, S.V., Jiang, D., Zhang, Y., Dubonos, S.V., Grigorieva, I.V., Firsov, A.A., 2004. Electric field effect in atomically thin carbon films. Science 306 (5696), 666-669. https://doi.org/10.1126/science.1102896.

Park, S., Ruoff, R.S., 2009. Chemical methods for the production of graphenes. Nat. Nanotechnol. 4, 217-224. https://doi.org/10.1038/nnano.2009.58.

Patil, R.U., Mishra, B.K., Singh, I.V., 2017. A new multiscale XFEM for the elastic properties evaluation of heterogeneous materials. Int. J. Mech. Sci. 122, 277-287. https://doi.org/10.1016/j.ijmecsci.2017.01.028.

Pingaro, Marco, Reccia, E., Trovalusci, P., 2019a. Homogenization of random porous materials with low-order virtual elements. ASCE-ASME J. Risk Uncertain. Eng. Syst. Part B Mech. Eng. 5 (3) https://doi.org/10.1115/1.4043475.

Pingaro, M., Reccia, E., Trovalusci, P., Masiani, R., 2019b. Fast statistical homogenization procedure (FSHP) for particle random composites using virtual element method. Comput. Mech. 64, 197-210. https://doi.org/10.1007/s00466 018-1665-7.

Qin, B., Wang, Q., Zhong, R., Zhao, X., Shuai, C., 2020. A three-dimensional solution for free vibration of FGP-GPLRC cylindrical shells resting on elastic foundations: a comparative and parametric study. Int. J. Mech. Sci. 187, 105896. https://doi.org/ 10.1016/j.ijmecsci.2020.105896.

Rafiee, M.A., Rafiee, J., Srivastava, I., Wang, Z., Song, H., Yu, Z.Z., Koratkar, N., 2010. Fracture and fatigue in graphene nanocomposites. Small 6 (2), 179-183. https://doi. org/10.1002/smll.200901480.

Rafiee, M.A., Rafiee, J., Wang, Z., Song, H., Yu, Z.Z., Koratkar, N., 2009. Enhanced mechanical properties of nanocomposites at low graphene content. ACS Nano 3, 3884-3890. https://doi.org/10.1021/nn9010472.

Reuss, A., 1929. Berechnung der Fließgrenze von Mischkristallen auf Grund der Plastizitätsbedingung für Einkristalle. ZAMM - Journal of Applied Mathematics and Mechanics/Z. Angew. Math. Mech. 9, 49-58. https://doi.org/10.1002/ zamm.19290090104.

Rodríguez-Ramos, R., de Medeiros, R., Guinovart-Díaz, R., Bravo-Castillero, J., Otero, J. A., Tita, V., 2013. Different approaches for calculating the effective elastic properties in composite materials under imperfect contact adherence. Compos. Struct. 99, 264-275. https://doi.org/10.1016/j.compstruct.2012.11.040.

Shin, H., Yang, S., Chang, S., Yu, S., Cho, M., 2013. Multiscale homogenization modeling for thermal transport properties of polymer nanocomposites with Kapitza thermal resistance. Polymer 54, 1543-1554. https://doi.org/10.1016/j. polymer.2013.01.020.

Shingare, K.B., Kundalwal, S.I., 2019a. Static and dynamic response of graphene nanocomposite plates with flexoelectric effect. Mech. Mater. 134, 69-84. https:// doi.org/10.1016/j.mechmat.2019.04.006.

Shingare, K.B., Kundalwal, S.I., 2020. Flexoelectric and surface effects on the electromechanical behavior of graphene-based nanobeams. Appl. Math. Model. 81, 70-91. https://doi.org/10.1016/j.apm.2019.12.021.

Smith, W.A., Auld, B.A., 1991. Modeling 1-3 composite piezoelectric: thickness-mode oscillations. IEEE Trans. Ultrason. Ferroelectrics Freq. Contr. 38, 40-47. https://doi. org/10.1109/58.67833.

Sun, W., Tzeng, J.T., 2002. Effective mechanical properties of EM composite conductors: an analytical and finite element modeling approach. Compos. Struct. 58, 411-421. https://doi.org/10.1016/S0263-8223(02)00129-0.

Suquet, P.M., 1987. Part IV: elements of homogenization for inelastic solid mechanics. In: Lecture Notes in Physics, vol. 272. Homogenization Techniques for Composite Media, p. 193. https://doi.org/10.1038/72256.

Tandon, G.P., Weng, G.J., 1984. The effect of aspect ratio of inclusions on the elastic properties of unidirectionally aligned composites. Polym. Compos. 5 (4), 327-333. https://doi.org/10.1002/pc.750050413.

Tang, L.C., Wan, Y.J., Yan, D., Pei, Y.B., Zhao, L., Li, Y.B., Wu, L. Bin, Jiang, J.X., Lai, G. Q., 2013. The effect of graphene dispersion on the mechanical properties of graphene/epoxy composites. Carbon 60, 16-27. https://doi.org/10.1016/j. carbon.2013.03.050.

Tian, W., Qi, L., Zhou, J., Liang, J., Ma, Y., 2015. Representative volume element for composites reinforced by spatially randomly distributed discontinuous fibers and its applications. Compos. Struct. 131, 366-373. https://doi.org/10.1016/j. compstruct.2015.05.014.

Voigt, W., 1910. Lehrbuch der kristallphysik:(mit ausschluss der kristalloptik), 34. BG Teubner. 
Wacker, G., Bledzki, A.K., Chate, A., 1998. Effect of interphase on the transverse Young's modulus of glass/epoxy composites. Compos. Part A Appl. Sci. Manuf. 29 (5-6), 619-626. https://doi.org/10.1016/S1359-835X(97)00116-4.

Wan, H., Delale, F., Shen, L., 2005. Effect of CNT length and CNT-matrix interphase in carbon nanotube (CNT) reinforced composites. Mech. Res. Commun. 32 (5), 481-489. https://doi.org/10.1016/j.mechrescom.2004.10.011.

Woodward, B., Kashtalyan, M., 2011. Three-dimensional elasticity solution for bending of transversely isotropic functionally graded plates. Eur. J. Mech. A Solids 30 (5), 705-718. https://doi.org/10.1016/j.euromechsol.2011.04.003.

Wu, H., Yang, J., Kitipornchai, S., 2017. Dynamic instability of functionally graded multilayer graphene nanocomposite beams in thermal environment. Compos. Struct. 162, 244-254. https://doi.org/10.1016/j.compstruct.2016.12.001.

Yamaguchi, S., Inoue, S., Sakai, T., Abe, T., Kitagawa, H., Imazato, S., 2017. Multi-scale analysis of the effect of nano-filler particle diameter on the physical properties of CAD/CAM composite resin blocks. Comput. Methods Biomech. Biomed. Eng. 20, 714-719. https://doi.org/10.1080/10255842.2017.1293664.

Yang, Q.S., Qin, Q.H., 2001. Fiber interactions and effective elasto-plastic properties of short-fiber composites. Compos. Struct. 54, 523-528. https://doi.org/10.1016/ S0263-8223(01)00129-5.

Young, R.J., Kinloch, I.A., Gong, L., Novoselov, K.S., 2012. The mechanics of graphene nanocomposites: a review. Compos. Sci. Technol. 72, 1459-1476. https://doi.org/ 10.1016/j.compscitech.2012.05.005.
Young, R.J., Lovell, P.A., 2011. Introduction to Polymers. CRC press. https://doi.org/ $10.1201 / 9781439894156$.

Zaman, I., Kuan, H.C., Dai, J., Kawashima, N., Michelmore, A., Sovi, A., Dong, S., Luong, L., Ma, J., 2012. From carbon nanotubes and silicate layers to graphene platelets for polymer nanocomposites. Nanoscale 4, 4578-4586. https://doi.org/ 10.1039/c2nr30837a.

Zaman, I., Phan, T.T., Kuan, H.C., Meng, Q., Bao La, L.T., Luong, L., Youssf, O., Ma, J., 2011. Epoxy/graphene platelets nanocomposites with two levels of interface strength. Polymer 52, 1603-1611. https://doi.org/10.1016/j.polymer.2011.02.003.

Zhang, Z., Li, Y., Wu, Helong, Zhang, H., Wu, Huaping, 2020. Mechanical analysis of functionally graded graphene oxide-reinforced composite beams based on the firstorder shear deformation theory. Mech. Adv. Mater. Struct. 27 (1), 3-11. https://doi. org/10.1080/15376494.2018.1444216.

Zhou, G., Sun, Q., Li, D., Meng, Z., Peng, Y., Chene, Z., Zeng, D., Su, X., 2020. Meso-scale modeling and damage analysis of carbon/epoxy woven fabric composite under Inplane tension and compression loadings. Int. J. Mech. Sci. 105980. https://doi.org/ 10.1016/j.ijmecsci.2020.105980.

Zhu, W., Chen, N., Liu, J., Xia, S., 2018. The effective elastic properties analysis of periodic microstructure with hybrid uncertain parameters. Int. J. Mech. Sci. 148, 39-49. https://doi.org/10.1016/j.ijmecsci.2018.08.018. 